\title{
S-Type and P-Type Habitability in Stellar Binary Systems: A Comprehensive Approach I. Method and Applications
}

\author{
M. Cuntz \\ Department of Physics \\ University of Texas at Arlington, Arlington, TX 76019-0059; \\ cuntz@uta.edu
}

\begin{abstract}
A comprehensive approach is provided to the study of both S-type and P-type habitability in stellar binary systems, which in principle can also be expanded to systems of higher order. P-type orbits occur when the planet orbits both binary components, whereas in case of S-type orbits the planet orbits only one of the binary components with the second component considered a perturbator. The selected approach encapsulates a variety of different aspects, which include: (1) The consideration of a joint constraint including orbital stability and a habitable region for a putative system planet through the stellar radiative energy fluxes ("radiative habitable zone"; RHZ) needs to be met. (2) The treatment of conservative, general and extended zones of habitability for the various systems as defined for the Solar System and beyond. (3) The providing of a combined formalism for the assessment of both S-type and P-type habitability; in particular, mathematical criteria are presented for which kind of system S-type and P-type habitability is realized. (4) Applications of the attained theoretical approach to standard (theoretical) main-sequence stars. In principle, five different cases of habitability are identified, which are: S-type and P-type habitability provided by the full extent of the RHZs; habitability, where the RHZs are truncated by the additional constraint of planetary orbital stability (referred to as ST and PT-type, respectively); and cases of no habitability at all. Regarding the treatment of planetary orbital stability, we utilize the formulae of Holman \& Wiegert (1999) [AJ 117, 621] as also used in previous studies. In this work we focus on binary systems in circular orbits. Future applications will also consider binary systems in elliptical orbits and provide thorough comparisons to other methods and results given in the literature.
\end{abstract}


Subject headings: astrobiology — binaries: general — celestial mechanics — planetary systems

\section{Introduction}

Starting more than a decade ago considerable observational evidence has been obtained indicating that planets are able to exist in stellar binary (and higher order) systems; see results and discussions by, e.g., Patience et al. (2002), Eggenberger et al. (2004), and Eggenberger et al. (2007). These observations are in line with the empirical finding that binary (and higher order) systems occur in high frequency in the local Galactic neighborhood (Duquennoy \& Mayor 1991; Lada 2006; Raghavan et al. 2006; Bonavita \& Desidera 2007; Raghavan et al. 2010). For example, Raghavan et al. (2010) presented results of a detailed analysis of companions to solar-type stars, based on a sample size of 454, and concluded that the overall fractions of double and triple systems are about $33 \%$ and $8 \%$, respectively, if all confirmed stellar and brown dwarf companions are accounted for. Updated results were meanwhile given by Roell et al. (2012). This study shows that 57 exoplanet host stars are identified having a stellar companion.

The fairly frequent occurrence of planets in binary systems is furthermore consistent with the presence of debris disks in a considerably large number of main-sequence star binary systems (e.g., Trilling et al. 2007). In principle, as discussed by Perryman (2011), planets in binary systems can be identified through two different venues: First, binaries or multiple star systems can be surveyed for the presence of planets by utilizing the established detection methods. Second, stars with detected planets can be scrutinized afterward to check if they possess one or more widely separated stellar companion(s); in this case, the planet(s) will also be categorized as belonging to a binary (or higher order) system.

From the view point of orbital mechanics, there are two different kinds of possible orbits (notwithstanding positions near the Lagrangian points $\mathrm{L}_{4}$ and $\mathrm{L}_{5}$ ) for planets in binary systems: S-type and P-type orbits (Dvorak 1982). A P-type orbit is given when the planet orbits both binary components, whereas in case of an S-type orbit the planet orbits only one of the binary components with the second component behaving as a perturbator. Eggenberger et al. (2004) presented a list of 15 planet-bearing binary systems with all planets in S-type orbits. They constitute mostly wide binaries with separation distances of up to 6400 AU; however, smaller separation distances on the order of 20 AU or less have also been identified. In the meantime, systems with planets in P-type orbits have also been identified. Arguably, 
the most prominent case is Kepler-16, as reported by Doyle et al. (2011) and previously suggested by Slawson et al. (2011), containing a Saturnian mass circumbinary planet. Quarles et al. (2012) have subsequently studied this system regarding the possibility of habitable exoplanets and habitable exomoons. Recently, a transiting circumbinary multiplanet system, i.e., Kepler-47, has also been identified (Orosz et al. 2012).

There is a significant body of literature devoted to the study of habitability ${ }^{1}$ in binary systems as well as in multiplanetary systems, which often also encompass stellar evolutionary considerations. Examples include the work by Jones et al. (2001), Noble et al. (2002), Menou \& Tabachnik (2003), Asghari et al. (2004), Sándor et al. (2007), Takeda et al. (2008), Dvorak et al. (2010), Jones \& Sleep (2010), and Kopparapu \& Barnes (2010). An important aspect that has received increased recognition in the literature is that in order for habitability to exist and to be maintained, a joint constraint that includes both orbital stability and a habitable environment for a system planet through the stellar radiative energy fluxes needs to be met. In the framework of this paper, the zone related to this latter requirement will subsequently be referred to as radiative habitable zone (RHZ), which constitutes a necessary, though often insufficient, condition for the existence of circumstellar habitability.

Previous work, mostly concentrated on the existence of habitability in single star multiplanetary systems, rendered the publication of detailed "stability catalogs" for the habitability zones of extrasolar planetary systems (e.g., Menou \& Tabachnik 2003; Sándor et al. 2007; Takeda et al. 2008; Dvorak et al. 2010; Kopparapu \& Barnes 2010). For example, Menou \& Tabachnik (2003) quantified the dynamical habitability of 85 planetary systems by considering the perturbing influence of giant planets beyond the traditional Hill sphere for close encounters with the theoretical terrestrial planets. They concluded that a significant fraction of the identified extrasolar planetary systems are unable to harbor habitable terrestrial planets. A statistical study on the stability of Earth-mass planets orbiting solar-mass

${ }^{1}$ The notion of habitability adopted in this study follows the conventional concept of Kasting et al. (1993) and related work, where habitability is defined based on the principal possibility that liquid water is able to exist on the surface of an Earth-type planet possessing a $\mathrm{CO}_{2} / \mathrm{H}_{2} \mathrm{O} / \mathrm{N}_{2}$ atmosphere (see Sect. 2 for details). More sophisticated approaches to habitability have been given in the meantime taking into account additional aspects, such as the planet's size and mass, atmospheric structure and composition, magnetic field, geodynamic properties, ionizing stellar UV and X-ray fluxes, and tidal locking (if existing) (e.g., Kasting \& Catling 2003, Scalo et al. 2007; Tarter et al. 2007; Zahnle et al. 2007, Selsis et al. 2008; Lammer et al. 2009, 2010; Kaltenegger et al. 2010; Horner \& Jones 2010, Cuntz et al. 2012; Forget 2013, Lucarini et al. 2013). Additionally, as pointed out by Williams \& Pollard (2002), planets with sufficiently thick atmospheres may remain habitable even when temporarily absent from their HZs due to orbits of considerable ellipticity. This possibility is disregarded in the following as well, as planets will be required to stay permanently in the CHZ, GHZ, or EHZ (see Sect. 2 for definitions), as applicable, to be considered habitable. 
stars in presence of stellar companions focusing on both the statistical properties of ejection times and the general prospects of planetary habitability was given by Fatuzzo et al. (2006). Additional work providing stability assessments for various observed extrasolar planetary systems based on detailed stability maps was given by Sándor et al. (2007). Takeda et al. (2008) explored the orbital stability or planets in double-planet systems for binaries by supplying an analytic framework based on secular perturbation theory; they also provided dynamical classification categories. Additional stability analyses to assess the habitability of planetary systems based on detailed numerical simulations were given by Dvorak et al. (2010) and Kopparapu \& Barnes (2010); note that the study of Dvorak et al. (2010) also dealt with a limited cases of planets in double star systems in orbit either around one stellar component (S-type) or around both components (P-type).

The study of planetary dynamics and habitable planet formation has meanwhile been described by, e.g., Quintana \& Lissauer (2010) and Haghighipour et al. (2010). They show that Earth-mass planets are, in principle, able to form in stellar binary systems, although many details of the relevant processes are not fully understood. The overarching conclusion of those investigations is that habitable planets in stellar binary (and, as anticipated, in higher-order systems) are, in general, possible, which is a stark motivation for providing a comprehensive study of S-type and P-type habitability in binary systems. The approach adopted in this study will be entirely analytic. Specifically, it will consider both S-type and P-type habitable orbits in the view of the joint constraint including orbital stability and a habitable region for a system planet through the appropriate amount of the stellar radiative energy fluxes. In an earlier study, Eggl et al. (2012) focused on S-type habitability in binary systems taking into account both circular and elliptical orbits for the stellar binary components; this latter aspect is however beyond the scope of the present work as we solely focus on systems in circular orbits. Numerical studies for P-type habitable environments with applications to Kepler-16, Kepler-34, Kepler-35 and Kepler-47 have been given by Kane \& Hinkel (2013).

Our paper is structured as follows: In Sect. 2, we comment on the adopted main-sequence star parameters and single star habitability. In Sect. 3, we introduce our theoretical approach suitable for stellar systems of the order of $N$, although our focus will be on binary systems. In this regard both S-type and P-type orbits will be examined, and detailed mathematical criteria for the existence of S-type and P-type RHZs will be derived. In Sect. 4, we consider the additional constraint of planetary orbital stability for the establishment of circumstellar habitability. Applications regarding S-type and P-type systems are given in Sect. 5, whereas the habitability classifications S, P, ST, and PT are introduced in Sect. 6. Section 7 conveys our summary and conclusions. 


\section{Stellar Parameters and Single Star Habitability}

In this study, S-type and P-type habitability is investigated mostly pertaining to standard (i.e., theoretical) main-sequence stars. The adopted stellar parameters, which are the stellar effective temperatures $T_{\text {eff }}$, the stellar radii $R_{*}$ (which together allow to define the stellar luminosities $L_{*}$ ), and the stellar masses $M_{*}$ are mainly based on the work by Gray (2005) (see his Table B.1) that assumes detailed photospheric spectral analyses. For stellar spectral types with no data available, the missing data were computed by employing biparabolic interpolation.

The exception, however, are data for stars of spectral type K5 V and below. In this regard we relied on the results from the spectral models of R. L. Kurucz and collaborators. They took into account hundreds of millions of spectral lines for a large set of atoms and molecules; see Castelli \& Kurucz (2004) and Kurucz (2005) for details. The effective temperatures implied by these models are in close similarity to those given by Gray (2005) for most types of stars; however, Gray (2005) reports consistently higher effective temperatures for stars of spectral type late-K and M; for the latter, the difference amounts to nearly $300 \mathrm{~K}$. Table 1 depicts the stellar parameters adopted for the present work.

An alternative approach expected to provide very similar results for either the stellar luminosity or the stellar mass (with the other parameter taken as fixed) is the employment of a mass-luminosity relationships applicable to main-sequence stars. The work by Reid (1987), as well as data from subsequent studies, yield

$$
\frac{L_{*}}{L_{\odot}}=\eta\left(\frac{M_{*}}{M_{\odot}}\right)^{\alpha}
$$

with $\eta=0.23$ and $\alpha=2.3$ for $M_{*}<0.43 M_{\odot}$ and $\eta=1$ and $\alpha=4.0$ for $M_{*} \geq 0.43 M_{\odot}$. At the high-mass end, this relationship holds until about $M_{*}=2 M_{\odot}$. It also becomes increasingly inaccurate for low-mass $\mathrm{M}$ dwarfs. Fortunately, the domains of applicability for Eq. (1) is consistent with most studies of binary habitability; see, e.g., Eggl et al. (2012) as example.

Next we focus on single star habitability, i.e., the evaluation of various limits of habitable zones $(\mathrm{HZs})$, which in the solar case shall be referred to as $s_{\ell}$. Previous work by, e.g., Kasting

et al. (1993) distinguished between conservative (CHZ) and the generalized habitable zone (GHZ), which can also be evaluated for general main-sequence stars, and other types of stars as well. For the Sun, the limits of the CHZ are given as 0.95 and $1.37 \mathrm{AU}(\ell=2$ and 4 , respectively), whereas for the GHZ, they are given as 0.84 and $1.67 \mathrm{AU}(\ell=1$ and 5 , respectively); see Table 2 .

The physical significance of the various kinds of HZs obtained by Kasting et al. (1993) 
can be summarized as follows: The GHZ is defined as bordered by the runaway greenhouse effect (inner limit) and the maximum greenhouse effect (outer limit). Concerning the latter it is assumed that a cloud-free $\mathrm{CO}_{2}$ atmosphere is still able to provide a surface temperature of $273 \mathrm{~K}$. The inner limit of the CHZ is defined by the onset of water loss. In this case, a wet stratosphere is assumed to exist where water is lost by photodissociation and subsequent hydrogen escape to space. Furthermore, the outer limit of the CHZ is defined by the first $\mathrm{CO}_{2}$ condensation attained by the onset of formation of $\mathrm{CO}_{2}$ clouds at a temperature of 273 K; see, e.g., Underwood et al. (2003) and Selsis et al. (2007) for further details. Table 3 conveys the results for the HZs for the different types of main-sequence stars of the present study with the different limits referred to as $\operatorname{HZ}\left(s_{\ell}\right)$.

For the outer edge of circumstellar habitability, even less stringent limits have been introduced in the meantime (e.g., Forget \& Pierrehumbert 1997; Mischna et al. 2000). They are based on the assumption of relatively thick planetary $\mathrm{CO}_{2}$ atmospheres as well as strong backwarming that may further be enhanced by $\mathrm{CO}_{2}$ crystals and clouds. These limits, which in case of the Sun correspond to $2.4 \mathrm{AU}\left(s_{\ell}=s_{6}\right)$, conform to the extended habitable zone (EHZ), have also been taken into account in our study, although the significance of the EHZ has meanwhile been criticized as a result of detailed planetary radiative transfer models (Halevy et al. 2009). Moreover, in the framework of the present study, we also consider planetary Earth-equivalent positions defined as $R_{\oplus \text {,eqv }} \simeq \sqrt{L_{*} / L_{\odot}}$ and labelled as $s_{\ell}=s_{3}$; see Table 3. It is meant as an intriguing reference distance of habitability both regarding single stars and stellar binary systems.

\section{Theoretical Approach}

\subsection{Basic Equations}

Next we introduce the governing equations for investigating the RHZs of binary systems pertaining to both S-type and P-type orbits. This approach targets the requirement of providing a habitable region for a system planet based on the radiative energy fluxes of the stellar components. The requirement of planetary orbital stability will be disregarded for now; it will be revisited in Sect. 4. The importance of orbital stability for allowing circumstellar habitability in stellar binaries will, however, be considered in an appropriate and consistent manner in the main body of the study.

For a star of luminosity $L_{i}$, given in units of solar luminosity $L_{\odot}$, the distance $d_{i}$ of the habitability limit $s_{\ell}$ as identified for the Sun, which may constitute either an inner or outer 
limit of habitability (except $\ell=3$ ), is given as

$$
d_{i}=s_{\ell} \sqrt{\frac{L_{i}}{S_{\mathrm{rel}, i \ell} L_{\odot}}}
$$

In case of a multiple star system of order $N$ with distances $d_{i}$, the limit of habitability related to $s_{\ell}$ is given as $2^{2}$

$$
\sum_{i=1}^{N} \frac{L_{i}}{S_{\mathrm{rel}, i \ell} d_{i}^{2}}=\frac{L_{\odot}}{s_{\ell}^{2}}
$$

In Eq. (2) and (3), $S_{\text {rel, }, i \ell}=S_{\text {rel }, i \ell}\left(T_{\text {eff }}\right)$ (see Table 1) describes the stellar flux in units of the solar constant that is a function of the stellar effective temperature $T_{\text {eff }}$ (e.g., Kasting et al. 1993; Underwood et al. 2003). Specifically, using the formalism[3 by Selsis et al. (2007), we find that

$$
S_{\mathrm{rel}, i \ell}=\left(\frac{s_{\ell}}{s_{\ell}-a_{z} T_{*}-b_{z} T_{*}^{2}}\right)^{2}
$$

with $s_{\ell}, a_{z}$, and $b_{z}$ in $\mathrm{AU}$, and $T_{*}=T_{\text {eff }}-5700$ in K. Selsis et al. (2007) also found that for $s_{\ell}<1$, corresponding to inner limits of habitability, the fitting parameters are given as $a_{z}=2.7619 \times 10^{-5}$ and $b_{z}=3.8095 \times 10^{-9}$, whereas for $s_{\ell}>1$, corresponding to outer limits of habitability, they are given as $a_{z}=1.3786 \times 10^{-4}$ and $b_{z}=1.4286 \times 10^{-9}$; note that $s_{\ell} \equiv 1$ corresponds to the customary notion of Earth-equivalent positions. Appropriate values for $s_{\ell}$ are given in Table 2 .

In the following we will focus on the case of binary systems, i.e., $N=2$. In this case

\footnotetext{
2 This equation is analogous to an equivalent equation of electrostatics relating a general distribution of charges to the resulting electrostatic potential in free space (Jackson 1999, see p. 40, Eq. (1.48)); a modified version of Eq. (3) has previously been considered by, e.g., Eggl et al. (2012).

${ }^{3} S_{\text {rel }, i \ell}$ represents the normalized stellar flux in units of the solar constant, $1368 \mathrm{~W} \mathrm{~m}^{-2}$, given by the stellar spectral energy distribution. Therefore, ordinarily, no $s_{\ell}$ dependence for $S_{\text {rel }, i \ell}$ should exist. However, the formulae by Selsis et al. (2007) utilize previous results by Kasting et al. (1993) who provided numerical values for limits of habitability for different types of stars considering various limit definitions (i.e., $s_{\ell}$ values identified for the Sun). But Kasting et al. (1993) used for the solar effective temperature an unusually low value of $5700 \mathrm{~K}$ instead of $5777 \mathrm{~K}$ as currently accepted (e.g., Stix 2004 ). Hence, transforming the polynomial fit based on the work by Kasting et al. aimed at considering the correct solar effective temperature renders a weak dependence on $s_{\ell}$ for the $S_{\text {rel }, i \ell}$ values. In contrast, the method by Underwood et al. (2003) provides a polynomial fit for $S_{\text {rel, } i \ell}$ without considering the solar temperature revision. An alternative method has been used by Cuntz \& Yeager (2009) and subsequent work. In this approach the polynomial fit by Underwood et al. (2003) is corrected via a triangular function based on data for stars of spectral type F0 V, G0 V, and $\mathrm{K} 0 \mathrm{~V}$. As a result the corresponding $S_{\text {rel, }, i \ell}$ values do also not depend on $s_{\ell}$.
} 
Eq. (3) reads

$$
\frac{L_{1}}{S_{\mathrm{rel}, 1 \ell} d_{1}^{2}}+\frac{L_{2}}{S_{\mathrm{rel}, 2 \ell} d_{2}^{2}}=\frac{L_{\odot}}{s_{\ell}^{2}}
$$

with

$$
\begin{aligned}
& d_{1}^{2}=a^{2}+z^{2}+2 a z \cos \varphi \\
& d_{2}^{2}=a^{2}+z^{2}-2 a z \cos \varphi .
\end{aligned}
$$

Here $a$ denotes the semidistance of binary separation, $z$ the distance of a position at the habitability limit contour (which later on will be referred to as "radiative habitable limit", see below), and $\varphi$ the associated angle; see Fig. 1 for information on the coordinate set-up for both S-type and P-type orbits. We will also assume $L_{1} \geq L_{2}$ without loss of generality.

With $L_{i \ell}^{\prime}$ defined as

$$
L_{i \ell}^{\prime}=\frac{L_{i}}{L_{\odot} S_{\mathrm{rel}, i \ell}},
$$

henceforth referred to as recast stellar luminosity (see Table 4), $z(\varphi)$ is given as

$$
z^{4}+A_{2} z^{2}+A_{1} z+A_{0}=0
$$

with

$$
\begin{aligned}
& A_{2}=2 a^{2}\left(1-2 \cos ^{2} \varphi\right)-s_{\ell}^{2}\left(L_{1 \ell}^{\prime}+L_{2 \ell}^{\prime}\right) \\
& A_{1}=2 a s_{\ell}^{2} \cos \varphi\left(L_{1 \ell}^{\prime}-L_{2 \ell}^{\prime}\right) \\
& A_{0}=a^{4}-a^{2} s_{\ell}^{2}\left(L_{1 \ell}^{\prime}+L_{2 \ell}^{\prime}\right) .
\end{aligned}
$$

Equation (8) constitutes a fourth-order algebraic equation that is known to possess four possible solutions (Bronshtein \& Semendyayev 1997), although some (or all) of them may constitute unphysical solutions, i.e., $z(\varphi)$ having a complex or imaginary value. The adopted coordinate system constitutes, in essence, a polar coordinate system except that negative values for $z$ are permitted; in this case the position of $z$ is found on the opposite side of angle $\varphi$.

In principle, it is possible to consider for Eq. (8) to only have solutions for $z(\varphi)$ given as $z \geq 0$; in this case the entire interval for $z(\varphi)$, which is $0 \leq \varphi<2 \pi$, needs to be examined. The following types of solutions are identified: For S-type orbits, two solutions exist in the intervals centered at $\varphi=0$ and at $\varphi=\pi$ (or one coinciding solution at each tangential point); see Fig. 1. However, there will be no solution in the typically relatively large intervals containing $\varphi=\pi / 2$ and $\varphi=3 \pi / 2$. Clearly, the size of any of those intervals 
critically depends on the system parameters $a, s_{\ell}, L_{1 \ell}^{\prime}$, and $L_{2 \ell}^{\prime}$, as expected. For P-type orbits, on the other hand, there will be one solution for each value of $\varphi$ in the range of $0 \leq \varphi<2 \pi$.

However, in general, negative values for the solutions of $z(\varphi)$ also exist. If taken into account, it will be sufficient to restrict the evaluation of Eq. (8) to the range $0 \leq \varphi \leq \pi$. In this case, for S-type orbits, there will be four solutions in the intervals with endpoints $\varphi=0$ and $\varphi=\pi$, as well as two solutions (if $L_{1 \ell}^{\prime} \neq L_{2 \ell}^{\prime}$ ) in a more extended interval containing these points. Also, a pair of solutions will become one coinciding solution at each tangential point. However, again, there will be no solution in the interval containing $\varphi=\pi / 2$. In case of P-type orbits, there will be two solutions for any value of $\varphi$ in the range of $0 \leq \varphi \leq \pi$. We will revisit this assessment in conjunction with the algebraic method for attaining the solution $z_{i}$; additionally, detailed mathematical criteria will be given for the existence of RHZ for S-type and P-type orbits.

Next we will focus on equal-star binary systems. Detailed solutions for general binary systems (i.e., systems of stellar components with by default unequal masses, luminosities, and effective temperatures) pertaining to both S-type and P-type orbits will be given in Sect. 3.3. Both subsections will be aimed at deriving RHZs; see, e.g., Williams \& Pollard (2002) for general discussions on the role of RHZs for the attainment of habitability in star-planet systems. However, strictly speaking, they will deal with identifying radiative habitable limits (RHLs) connected to a distinct value of $s_{\ell}$ noting that manifesting a RHZ requires that the RHL for $s_{\ell \text {,out }}$ to be located completely outside of the RHL for $s_{\ell \text {,in }}$ with $s_{\ell, \text { in }}$ and $s_{\ell \text {,out }}$ appropriately paired. A summary about the existence and structure of the RHZs, encompassing the radiative CHZs, GHZs, and EHZs, will be given in Sect. 3.4; this subsection will also convey cases where no RHZs exist due to the behavior of the RHLs owing to the choices of $s_{\ell, \text { in }}$ and $s_{\ell, \text { out }}$.

\subsection{Equal-Star Binary Systems}

Now we focus on the special case of equal-star binary systems, i.e., stars of identical recast luminosities, i.e., $L_{1 \ell}^{\prime}=L_{2 \ell}^{\prime}=L_{\ell}^{\prime}$. For theoretical main-sequence stars this assumption also implies $S_{\text {rel, } 1 \ell}=S_{\text {rel, } 2 \ell}$ and $M_{1}=M_{2}$; this latter assumption about the stellar masses is relevant for the orbital stability constraint of system planets. With $A_{1}=0$, Eq. (8) now constitutes a biquadratic equation that can be solved in a straightforward manner. The other coefficients are given as

$$
A_{2}=2 a^{2}\left(1-2 \cos ^{2} \varphi\right)-2 s_{\ell}^{2} L_{\ell}^{\prime}
$$




$$
A_{0}=a^{4}-2 a^{2} s_{\ell}^{2} L_{\ell}^{\prime}
$$

Thus, the solution of Eq. (8) is given as

$$
z= \pm \sqrt{-a^{2}\left(1-2 \cos ^{2} \varphi\right)+s_{\ell}^{2} L_{\ell}^{\prime} \pm \sqrt{D_{2}}}
$$

with

$$
D_{2}=s_{\ell}^{4} L_{\ell}^{\prime 2}+4 a^{4} \cos ^{4} \varphi-4 a^{2} \cos ^{2} \varphi\left(a^{2}-s_{\ell}^{2} L_{\ell}^{\prime}\right) .
$$

With known systems parameters, which are $a, s_{\ell}$, and $L_{\ell}^{\prime}$, the function $z(\varphi)$, describing the habitability limits for the binary system associated with inner limit and outer limit values $s_{\ell}$ derived for the Sun (see Sect. 2 for details) can be obtained in a straightforward manner.

Owing to the system symmetry, the existence of S-type and P-type RHLs can be identified by attaining the solutions of Eq. (11) for $\varphi=0$ and $\varphi=\pi / 2$. First we examine the solutions of Eq. (11) for $\varphi=0$, i.e., $\cos \varphi=1$, which are given as

$$
z= \pm \sqrt{a^{2}+s_{\ell}^{2} L_{\ell}^{\prime} \pm s_{\ell} \sqrt{s_{\ell}^{2} L_{\ell}^{\prime 2}+4 a^{2} L_{\ell}^{\prime}}} .
$$

This allows us to explore the existence of S-type RHLs. The total number of solutions for $z_{i}$ (if existing) is four as expected, which can be ordered as $z_{1}<z_{2}<z_{3}<z_{4}$. Due to symmetry it is found that $z_{3} \geq 0$, which implies that

$$
a^{2}+s_{\ell}^{2} L_{\ell}^{\prime}-s_{\ell} \sqrt{s_{\ell}^{2} L_{\ell}^{2}+4 a^{2} L_{\ell}^{\prime}} \geq 0
$$

Thus, the condition for the existence of S-type RHLs is given as

$$
a \geq s_{\ell} \sqrt{2 L_{\ell}^{\prime}}
$$

Next we examine the solutions of Eq. (11) for $\varphi=\pi / 2$, i.e., $\cos \varphi=0$. This allows us to explore the existence of P-type RHLs; the latter implies two solutions of Eq. (11) regardless of the value for $\varphi$. If the positive root of $D_{2}$ (see Eq. 12) is considered, the solution is given as

$$
z= \pm \sqrt{-a^{2}+2 s_{\ell}^{2} L_{\ell}^{\prime}}
$$

Thus, the condition for the existence of P-type RHLs is given as

$$
a \leq s_{\ell} \sqrt{2 L_{\ell}^{\prime}}
$$

Therefore, Eqs. (15) and (17) allow to identify the conditions for S-type and P-type RHLs, respectively, for equal-star binary systems, which depend on the systems parameters 
$a, s_{\ell}$, and $L_{\ell}^{\prime}$; note that the equal signs in these equations carry little relevance. Comparing Eqs. (15) and (17) also implies that the joint existence of S-type and P-type habitability in equal-star binary systems in circular orbits is not possible, irrespectively of the system parameters and the planetary orbital stability requirement (see Sect. 4), noting that the latter imposes an additional constraint on habitability even when the RHZ-related conditions are met. Figure 2 depicts the borders of the S-type and P-type radiative habitable limits, i.e., RHLs, for different values of $s_{\ell}$ in regard to $a$ and $L_{\ell}^{\prime}$.

\subsection{General Binary Systems}

\subsubsection{Method of Solution}

We now focus on obtaining solutions for our key equation, Eq. (8), pertaining to S-type and P-type RHLs for general binary systems, i.e., $L_{1 \ell}^{\prime} \neq L_{2 \ell}^{\prime}$. Following, e.g., Abramowitz \& Stegun (1972) and Beyer (1987), the set of solutions for a fourth-order polynomial reads

$$
\begin{aligned}
z_{1} & =-\frac{1}{2} \mathcal{C}-\frac{1}{2} \mathcal{D} \\
z_{2} & =-\frac{1}{2} \mathcal{C}+\frac{1}{2} \mathcal{D} \\
z_{3} & =+\frac{1}{2} \mathcal{C}-\frac{1}{2} \mathcal{E} \\
z_{4} & =+\frac{1}{2} \mathcal{C}+\frac{1}{2} \mathcal{E}
\end{aligned}
$$

with

$$
\begin{aligned}
\mathcal{C} & =\sqrt{-2 a^{2}\left(1-2 \cos ^{2} \varphi\right)+s_{\ell}^{2}\left(L_{1 \ell}^{\prime}+L_{2 \ell}^{\prime}\right)+y_{1}} \\
\mathcal{D} & =\sqrt{s_{\ell}^{2}\left(L_{1 \ell}^{\prime}+L_{2 \ell}^{\prime}\right)+4 a s_{\ell}^{2}\left(L_{1 \ell}^{\prime}-L_{2 \ell}^{\prime}\right) \mathcal{C}^{-1} \cos \varphi-2 a^{2}\left(1-2 \cos ^{2} \varphi\right)-y_{1}} \\
\mathcal{E} & =\sqrt{s_{\ell}^{2}\left(L_{1 \ell}^{\prime}+L_{2 \ell}^{\prime}\right)-4 a s_{\ell}^{2}\left(L_{1 \ell}^{\prime}-L_{2 \ell}^{\prime}\right) \mathcal{C}^{-1} \cos \varphi-2 a^{2}\left(1-2 \cos ^{2} \varphi\right)-y_{1}}
\end{aligned}
$$

with $y_{1}$ as a solution of the resolvent cubic equation

$$
y^{3}-A_{2} y^{2}-A_{0} y+\left(4 A_{2} A_{0}-A_{1}^{2}\right)=0
$$

with $A_{0}, A_{1}$, and $A_{2}$ given by Eqs. (9a) to $(9 \mathrm{c})$; here the term of $\left(L_{1 \ell}^{\prime}-L_{2 \ell}^{\prime}\right) \mathcal{C}^{-1}$ in Eqs. (19b) and $(19 \mathrm{c})$ corresponds to the case of non-equal star binaries assumed in the following. Note that for equal-star binaries a more straightforward method of solution is available (see Sect. 3.2). The solutions $z_{i}$ given through Eqs. (18a) to (18d), if existing, are ordered as $z_{1}<$ 
$z_{2}<z_{3}<z_{4}$ for $\varphi=0$; this order is also maintained for any other value of $\varphi$ as identified in all model simulations pursued. Although Eq. (20) has three possible solutions, there is only one appropriate choice for $y$, named $y_{1}$, because it is necessary to avoid that all $z_{i}$ obtained through Eq. (8) are of imaginary or conjugate complex value in cases where S-type or P-type RHLs exist.

The acceptable solution for $y$ is given as

$$
y_{1}=-\frac{1}{3} \hat{a}_{2}+(S+T)
$$

with the substitutions

$$
\begin{aligned}
S & =\sqrt[3]{R+\sqrt{D_{3}}} \\
T & =\sqrt[3]{R-\sqrt{D_{3}}} \\
D_{3} & =Q^{3}+R^{2}
\end{aligned}
$$

and with $Q$ and $R$ given as

$$
\begin{aligned}
Q & =\frac{1}{3} \hat{a}_{1}-\frac{1}{9} \hat{a}_{2}^{2} \\
R & =-\frac{1}{2} \hat{a}_{0}+\frac{1}{6} \hat{a}_{1} \hat{a}_{2}-\frac{1}{27} \hat{a}_{2}^{3}
\end{aligned}
$$

while noting that

$$
\begin{aligned}
& \hat{a}_{0}=4 A_{0} A_{2}-A_{1}^{2} \\
& \hat{a}_{1}=-4 A_{0} \\
& \hat{a}_{2}=-A_{2} .
\end{aligned}
$$

These sets of equation can be solved and appropriate values for $z(\varphi)$ can be obtained. The results will depend on the system parameters $a, s_{\ell}, L_{1 \ell}^{\prime}$, and $L_{2 \ell}^{\prime}$, as expected.

Next we describe the solutions for S-type and P-type RHLs in more detail. It is important to recognize that a priori choices about the existence of S-type and P-type RHLs are

neither necessary nor possible as the existence of any of those RHLs is determined by the fulfillment of well-defined mathematical conditions; they will also be given in the following.

\subsubsection{S-type Orbits}

An analysis of the possible solutions for Eqs. (18a) to (18d) shows that for S-type RHLs valid solutions are obtained based on

$$
S=\sqrt[6]{R^{2}+K^{2}}(\cos \xi+i \sin \xi)
$$




$$
T=\sqrt[6]{R^{2}+K^{2}}(\cos \xi-i \sin \xi)
$$

with

$$
\begin{aligned}
K & =\sqrt{\left|D_{3}\right|} \\
\xi & =\frac{1}{3} \arctan \left(\frac{K}{R}\right)
\end{aligned}
$$

with $R$ given by Eq. (23b). Therefore, the solution of the resolvent cubic equation, Eq. (20), is given as

$$
y_{1}=-\frac{1}{3} \hat{a}_{2}+2 \sqrt[6]{R^{2}+K^{2}} \cos \xi
$$

For the values of $z_{i}$ it is found that for the interval centered at $\varphi=0, z_{1}$ and $z_{2}$ exhibit negative values, whereas $z_{3}$ and $z_{4}$ exhibit positive values with $z_{1}<z_{2}<z_{3}<z_{4}$. Thus, $z_{1}$ and $z_{2}$ describe the RHL regarding star S1, whereas $z_{3}$ and $z_{4}$ describe the RHL regarding star S2 (see Fig. 1). Conversely, for the interval centered at $\varphi=\pi, z_{1}$ and $z_{2}$ again exhibit negative values and $z_{3}$ and $z_{4}$ exhibit positive values. In this case, $z_{1}$ and $z_{2}$ describe the RHL for star S2, whereas $z_{3}$ and $z_{4}$ describe the RHL for star S1. No solutions are obtained in the vicinity of $\varphi=\pi / 2$ and $\varphi=3 \pi / 2$, as expected. Thus, for each angle $\varphi$ in the range of $0 \leq \varphi<2 \pi$ the appropriate number of solutions is attained to describe S-type RHLs. However, due to symmetry, solutions are only needed for $0 \leq \varphi \leq \pi$.

The existence of S-type RHLs requires that $z_{2} \leq z_{3}$ because otherwise the two distinct S-type RHL contours about the two binary components would not be separated, which corresponds to the condition

$$
\mathcal{C} \geq \frac{1}{2}(\mathcal{D}+\mathcal{E}) .
$$

Equation (28) can be rewritten to provide an expression based on the system parameters $A_{0}, A_{1}$, and $A_{2}$ defined through Eqs. (9a) to (9c). It is found that

$$
2 y_{1}\left(A_{2}-y_{1}\right)^{2}-A_{1}^{2} \geq 0
$$

with $y_{1}=y_{1}\left(A_{0}, A_{1}, A_{2}\right)$. The expression for $y_{1}$ is highly complicated; however, it can be obtained based on Eqs. (21) to (24c) by using, e.g., MATHEMATICA ${ }^{\circledR}$ in a straightforward manner.

In conclusion, for S-type RHLs to exist for the system parameters $a, s_{\ell}, L_{1 \ell}^{\prime}$, and $L_{2 \ell}^{\prime}$, it is necessary that the relations (28) and (29), which are equivalent, must be fulfilled for any angle of $\varphi$, though the evaluation can be limited to $\varphi=0$. Furthermore, through analytical transformations it can be shown that the condition depicted as Eqs. (28) and (29) requires

$$
6912 A_{0}^{3}-3456 A_{0}^{2} A_{2}^{2}+432 A_{0} A_{2}^{4}-729 A_{1}^{4} \leq 0 .
$$


In the limiting case of equal-star binary systems, attained as $A_{1} \rightarrow 0$, Eq. (30) can be simplified as

$$
\left(4 A_{0}-A_{2}^{2}\right)^{2} \geq 0
$$

this relationship is fulfilled in a trivial manner.

\subsubsection{P-type Orbits}

An analysis of the possible solutions for Eqs. (18a) to (18d) also shows that for P-type RHLs valid solutions require

$$
D_{3} \geq 0
$$

see Eq. (22c). The detailed evaluation of this condition requires the evaluation of various sets of equations denoted as Eqs. (9a) to (9c), (22a) to (22c), and (23a) to (23b); see Sect. 3.1 and 3.3.1.

In terms of the solutions for P-type RHLs it is found that for $0 \leq \varphi<\pi / 2$ and $3 \pi / 2<\varphi \leq 2 \pi, z_{1}$ exhibit negative values and $z_{2}$ exhibit positive values, whereas $z_{3}$ and $z_{4}$ are undefined; they are also not needed for outlining P-type RHLs. Moreover, for the range of $\pi / 2<\varphi<3 \pi / 2, z_{3}$ exhibit negative values and $z_{4}$ exhibit positive values, noting that $z_{1}$ and $z_{2}$ remain undefined. For $\pi / 2$ and $3 \pi / 2$, removable singularities are identified, which can easily be fixed through interpolation taking values of $z$ for neighboring angles of $\varphi$. In summary, for each angle $\varphi$ in the range of $0 \leq \varphi<2 \pi$ two values of $z_{i}$ (i.e., one positive and one negative value) are identified allowing to determine P-type RHLs. However, due to symmetry, solutions are only needed for $0 \leq \varphi \leq \pi$.

Moreover, through analytical transformations it can be shown that the condition depicted as Eq. (32) can be rewritten as

$$
16 A_{0} A_{2}^{4}+144 A_{0} A_{1}^{2} A_{2}-128 A_{0}^{2} A_{2}^{2}+256 A_{0}^{3}-4 A_{1}^{2} A_{2}^{3}-27 A_{1}^{4} \leq 0 .
$$

with $A_{0}, A_{1}$, and $A_{2}$ defined through Eqs. (9a) to (9c) with the left hand side of Eq. (33) representing $-108 \cdot D_{3}$. In conclusion, for P-type RHLs to exist for the system parameters $a$, $s_{\ell}, L_{1}^{\prime}$, and $L_{2}^{\prime}$, it is necessary that the relations (32) and (33), which are equivalent, must be fulfilled for any angle of $\varphi$, though the evaluation can be limited to $\varphi=\pi / 2$. In the limiting case of equal-star binary systems, attained as $A_{1} \rightarrow 0$, Eq. (33) can be simplified as

$$
\left(4 A_{0}-A_{2}^{2}\right)^{2} \geq 0
$$

this relationship, already given as Eq. (31), is fulfilled in a trivial manner. 


\subsection{Calculation of RHZs for Binary Systems}

The identification of the RHZs in binary systems requires the calculation of limits of habitable zones, i.e., RHLs, as pointed out in Sect. 3.1. The RHZs need to be established for values of $s_{\ell}$ with $\ell=1,2,4,5$, and 6 (see Sect. 2 and Table 2), which are informed by model-dependent physical limits of habitability for the solar environment (e.g., Kasting et al. 1993). As part of the process, the parameters of $s_{\ell}$ need to be appropriately paired in terms of the inner and outer limits of habitability. For the CHZ the parameters $\left(s_{\ell, \text { in }}, s_{\ell, \text { out }}\right)$ need to be paired as $\left(s_{2}, s_{4}\right)$, whereas for the GHZ, they need to paired as $\left(s_{1}, s_{5}\right)$. For the EHZ, the parameters of $s_{\ell}$ need to paired as $\left(s_{1}, s_{6}\right)$, considering that both the CHZ and the GHZ shall be viewed as subdomains of the EHZ.

For S-type and P-type orbits, the radiative zones of habitability $\mathrm{RHZ}(z)$, which constitutes a circular region (annulus) around each star S1 and S2 (S-type) or both stars (P-type) can be determined as

$$
\operatorname{RHZ}(z)=\left.\operatorname{Min}(\mathcal{R}(z, \alpha))\right|_{s_{\ell, \text { out }}}-\left.\operatorname{Max}(\mathcal{R}(z, \alpha))\right|_{s_{\ell, \text { in }}}
$$

and

$$
\operatorname{RHZ}(z)=\left.\operatorname{Min}(\mathcal{R}(z, \varphi))\right|_{s_{\ell, \text { out }}}-\left.\operatorname{Max}(\mathcal{R}(z, \varphi))\right|_{s_{\ell, \text { in }}},
$$

respectively; see Fig. 1 for coordinate information. Here $\mathcal{R}(z, \alpha)$ and $\mathcal{R}(z, \varphi)$ describe the areas bordered by the RHLs defined by $s_{\ell, \text { in }}$ and $s_{\ell \text {,out }}$. The calculation of the extrema is applied to the angles $\alpha$ and $\varphi$ for the intervals $0 \leq \alpha \leq \pi$ and $0 \leq \varphi \leq \pi / 2$, respectively; note that we assumed $L_{1} \geq L_{2}$ without loss of generality. In the S-type case the calculation of the extrema pertaining to the RHZ values is based on the angular coordinate $\alpha$ instead of $\varphi$; however, the angular coordinate $\varphi$ is still needed for the calculation of $z_{i}$ as part of the overall approach toward identifying S-type and P-type habitability.

Figure 3 depicts examples of RHLs and RHZs for different types of systems. In the Stype case the RHLs are bended toward the center of the system, whereas in the P-type case they are of notable elliptical shape. The RHZs always constitute circular annuli obtained through inspecting the appropriate minima and maxima of the RHLs. The examples as depicted include S-type and P-type systems with separation distances $2 a$ of $0.5 \mathrm{AU}$ and 5.0 AU, respectively. Cases of both equal-star and non-equal star binaries are selected. The focus of this figure is the identification of the appropriate circular region (i.e., annulus) for each case. The figure also indicates the portions within the $\mathcal{R}(z, \alpha)$ and $\mathcal{R}(z, \varphi)$ domains that are not part of the $\operatorname{RHZ}(z)$ annuli.

Next we determine the values for the extrema pertaining to $\operatorname{RHZ}(z)$ following Eqs. (35)

and (36) based on the solutions of Eq. (8) given as Eqs. (18a) to (18d). In cases where 
four solutions $z_{i}$ exist, it is found that they are ordered as $z_{1}<z_{2}<z_{3}<z_{4}$ with $z_{1}$ and $z_{2}$ constituting negative values, and $z_{3}$ and $z_{4}$ constituting positive values. If the negative solutions for $z_{i}$ are permitted, it is sufficient for S-type orbits, both for star S1 and S2, to only consider solutions for $\varphi=0$. For P-type orbits, a more detailed assessment is required (see below). For S-type orbits, regarding star S1, the extrema are obtained as

$$
\begin{aligned}
\mathrm{RHZ}_{\text {in }} & =\left.\operatorname{Max}(\mathcal{R}(z, \alpha))\right|_{s_{\ell, \text { in }}}=\left.\left|a+z_{2}(0)\right|\right|_{s_{\ell, \text { in }}} \\
\mathrm{RHZ}_{\text {out }} & =\left.\operatorname{Min}(\mathcal{R}(z, \alpha))\right|_{s_{\ell, \text { out }}}=\left.\left|a+z_{1}(0)\right|\right|_{s_{\ell, \text { out }}},
\end{aligned}
$$

and for star S2, they are obtained as

$$
\begin{aligned}
\mathrm{RHZ}_{\text {in }} & =\left.\operatorname{Max}(\mathcal{R}(z, \alpha))\right|_{s_{\ell, \mathrm{in}}}=\left.\left|a-z_{3}(0)\right|\right|_{s_{\ell, \text { in }}} \\
\mathrm{RHZ}_{\text {out }} & =\left.\operatorname{Min}(\mathcal{R}(z, \alpha))\right|_{s_{\ell, \mathrm{out}}}=\left.\left|a-z_{4}(0)\right|\right|_{s_{\ell, \mathrm{out}}} .
\end{aligned}
$$

The size of each annulus $\Delta_{\mathrm{RHZ}}$ for pairs $\left(s_{\ell, \text { in }}, s_{\ell, \text { out }}\right)$ is given as $\Delta_{\mathrm{RHZ}}=\mathrm{RHZ}$ out $-\mathrm{RHZ}_{\text {in }}$. $\mathrm{RHZ}_{\text {in }}$ also constitutes a generalization of $\mathrm{HZ}\left(s_{\ell}\right)$ with $\ell=1,2$ previously defined for single stars (see Sect. 2 and Table 3 ). Likewise, $\mathrm{RHZ}_{\text {out }}$ constitutes a generalization of $\mathrm{HZ}\left(s_{\ell}\right)$ with $\ell=4,5$, and 6 .

For P-type orbits, the extrema are given as follows:

$$
\begin{aligned}
\mathrm{RHZ}_{\text {in }} & =\left.\operatorname{Max}(\mathcal{R}(z, \varphi))\right|_{s_{\ell, \text { in }}}=\left.\left|z_{1}(0)\right|\right|_{s_{\ell, \text { in }}} \\
\mathrm{RHZ}_{\text {out }} & =\left.\operatorname{Min}(\mathcal{R}(z, \varphi))\right|_{s_{\ell, \text { out }}}=\left.\left|z_{1}\left(\varphi_{\text {out }}\right)\right|\right|_{s_{\ell, \text { out }}},
\end{aligned}
$$

We note that for the angle $\varphi_{\text {out }}$ for $\mathrm{RHZ}_{\text {out }}$ no straightforward expression $4^{4}$ exists; it is located in the interval $0<\varphi_{\text {out }} \leq \pi / 2$. It can be found numerically as it is given by the angle where the minimum of $|z(\varphi)|$ occurs. In the special case of $L_{2 \ell}^{\prime} / L_{1 \ell}^{\prime} \ll 1$ with $L_{1 \ell}^{\prime}$ and $L_{2 \ell}^{\prime}$ denoting the stellar primary and secondary, respectively, it is found that $\varphi_{\text {out }} \rightarrow 0$, whereas for $L_{1 \ell}^{\prime}=L_{2 \ell}^{\prime}$ it is found that $\varphi_{\text {out }}=\pi / 2$ (see Sect. 3.2).

There is also another complication in the identification of RHZ P-type orbits. Generally, it is required for the RHL of $s_{\ell \text {,out }}$ to be located completely outside of the RHL of $s_{\ell \text {,in }}$, i.e.,

$$
\left.\operatorname{Min}(\mathcal{R}(z, \varphi))\right|_{s_{\ell, \text { out }}} \geq\left.\operatorname{Max}(\mathcal{R}(z, \varphi))\right|_{s_{\ell, \text { in }}}
$$

\footnotetext{
${ }^{4}$ Due to the nature of the underlying equations for $z_{i}$, an analytic expression for $\varphi_{\text {out }}$ is deemed possible. However, it will be highly complicated and thus a numerical solution may be preferred.
} 
This condition is however violated in some models, especially for relatively large values of $a$ as well as relatively small ratios of $L_{2 \ell}^{\prime} / L_{1 \ell}^{\prime}$. In this case the RHZ for $\left(s_{\ell, \text { in }}, s_{\ell, \text { out }}\right)$ is nullified, a behavior that may occur for the pairings $\left(s_{2}, s_{4}\right),\left(s_{1}, s_{5}\right)$, and $\left(s_{1}, s_{6}\right)$, corresponding to the $\mathrm{CHZ}, \mathrm{GHZ}$, and EHZ, respectively. In this regard the existence of the CHZ is in most jeopardy as $\left(s_{4}-s_{2}\right)$ constitutes the smallest bracket among the various kinds of HZs (see Table 2). Also note that if the GHZ is nullified, the CHZ will be nullified as well considering that the CHZ (if existing) is entirely located within the GHZ. Likewise, if the EHZ is nullified the existence of both the CHZ and the GHZ will be nullified. Detailed examples will be given in the application segment of this paper; see Sect. 5.2 for details. However, this type of phenomenon does not occur for RHZs pertaining to S-type orbits.

For equal-star binary systems, with the property of $L_{1 \ell}^{\prime}=L_{2 \ell}^{\prime}=L_{\ell}^{\prime}$, expressions for $\mathrm{RHZ}_{\text {in }}$ and $\mathrm{RHZ}_{\text {out }}$ for S-type and P-type orbits can be obtained based on Eqs. (13) and (16). For S-type orbits we find

$$
\begin{aligned}
\mathrm{RHZ}_{\text {in }} & =\left|a-\sqrt{a^{2}+s_{\ell, \text { in }}^{2} L_{\ell, \text { in }}^{\prime}+s_{\ell, \text { in }} \sqrt{s_{\ell, \text { in }}^{2} L_{\ell, \text { in }}^{\prime 2}+4 a^{2} L_{\ell, \text { in }}^{\prime}}}\right| \\
\mathrm{RHZ}_{\text {out }} & =\left|a-\sqrt{a^{2}+s_{\ell, \text { out }}^{2} L_{\ell, \text { out }}^{\prime}-s_{\ell, \text { out }} \sqrt{s_{\ell, \text { out }}^{2} L_{\ell, \text { out }}^{\prime 2}+4 a^{2} L_{\ell, \text { out }}^{\prime}}}\right| .
\end{aligned}
$$

It is also intriguing to explore the limits $a \gg s_{\ell, \text { in }} \sqrt{L_{\ell, \text { in }}^{\prime}}$ and $a \gg s_{\ell, \text { out }} \sqrt{L_{\ell, \text { out }}^{\prime}}$. If these limits are met, it is found that

$$
\begin{aligned}
\mathrm{RHZ}_{\text {in }} & =\left|\frac{1}{2 a}\left(s_{\ell, \text { in }}^{2} L_{\ell, \text { in }}^{\prime}+s_{\ell, \text { in }} \sqrt{s_{\ell, \text { in }}^{2} L_{\ell, \text { in }}^{\prime 2}+4 a^{2} L_{\ell, \text { in }}^{\prime}}\right)\right| \\
\mathrm{RHZ}_{\text {out }} & =\left|\frac{1}{2 a}\left(s_{\ell, \text { out }}^{2} L_{\ell, \text { out }}^{\prime}-s_{\ell, \text { out }} \sqrt{s_{\ell, \text { out }}^{2} L_{\ell, \text { out }}^{\prime 2}+4 a^{2} L_{\ell, \text { out }}^{\prime}}\right)\right| .
\end{aligned}
$$

Moreover, in the limit of $a \rightarrow \infty$, the expressions for single star HZs regarding $\mathrm{RHZ}_{\text {in }}$ and $\mathrm{RHZ}_{\mathrm{out}}$ are recovered, as expected, which are given as $s_{\ell \text {,in }} \sqrt{L_{\ell, \text { in }}^{\prime}}$ and $s_{\ell, \text { out }} \sqrt{L_{\ell, \text { out }}^{\prime}}$, respectively. They are in agreement with the expressions previously obtained by Kasting et al. (1993), Underwood et al. (2003), Selsis et al. (2007), and others.

Results for P-type orbits can be obtained considering

$$
\begin{aligned}
\mathrm{RHZ}_{\text {in }} & =\left.\operatorname{Max}\left(\mathcal{R}(z, 0), \mathcal{R}\left(z, \frac{\pi}{2}\right)\right)\right|_{s_{\ell, \text { in }}}=\left.\mathcal{R}(z, 0)\right|_{s_{\ell, \text { in }}} \\
\mathrm{RHZ}_{\text {out }} & =\left.\operatorname{Min}\left(\mathcal{R}(z, 0), \mathcal{R}\left(z, \frac{\pi}{2}\right)\right)\right|_{s_{\ell, \mathrm{out}}}=\left.\mathcal{R}\left(z, \frac{\pi}{2}\right)\right|_{s_{\ell, \mathrm{out}}} .
\end{aligned}
$$

In this case we find

$$
\begin{aligned}
\mathrm{RHZ}_{\text {in }} & =\sqrt{a^{2}+s_{\ell, \text { in }}^{2} L_{\ell, \text { in }}^{\prime}+s_{\ell, \text { in }} \sqrt{s_{\ell, \text { in }}^{2} L_{\ell, \text { in }}^{\prime 2}+4 a^{2} L_{\ell, \text { in }}^{\prime}}} \\
\mathrm{RHZ}_{\text {out }} & =\sqrt{-a^{2}+2 s_{\ell, \text { out }}^{2} L_{\ell, \text { out }}^{\prime}}
\end{aligned}
$$


based on the system parameters $a, s_{\ell, \text { in }}, s_{\ell, \text { out }}, L_{\ell, \text { in }}^{\prime}$, and $L_{\ell, \text { out }}^{\prime}$. Additionally, the requirement to avoid that the RHL for $s_{\ell \text {,out }}$ to be partially or completely located inside of the RHL for $s_{\ell, \text { in }}$, see Eq. (40), entails

$$
a^{2}+\frac{1}{2} s_{\ell, \text { in }}^{2} L_{\ell, \text { in }}^{\prime}+s_{\ell, \text { in }} \sqrt{a^{2} L_{\ell, \text { in }}^{\prime}+\frac{1}{4} s_{\ell, \text { in }}^{2} L_{\ell, \text { in }}^{\prime 2}}-s_{\ell, \text { out }}^{2} L_{\ell, \text { out }}^{\prime} \leq 0,
$$

which allows to set constraints on the separation distance $2 a$ of the binary system noting that the values of $s_{\ell, \text { in }}, s_{\ell, \text { out }}, L_{\ell, \text { in }}^{\prime}$, and $L_{\ell, \text { out }}^{\prime}$ are subject to distinct restrictions, particularly in case of main-sequence stars (see Tables 1 and 2). Depictions of the condition (45) for equal-star binaries for the pairings $\left(s_{2}, s_{4}\right),\left(s_{1}, s_{5}\right)$, and $\left(s_{1}, s_{6}\right)$, corresponding to the CHZ, GHZ, and EHZ, respectively, are given in Fig. 4. A similar expression is expected to hold for nonequal-star binaries, albeit it will be highly complicated. Hence, for those systems a numerical assessment of $\mathrm{RHZ}_{\text {in }}$ and $\mathrm{RHZ}_{\text {out }}$ (see Eqs. 39a and 39b) may be preferred to accommodate condition (40); see Sect. 5.2.2 for additional information and data.

\section{Constraints on Habitability due to Planetary Orbital Stability}

A primary constraint on planetary habitability is that planets are required to exist in the HZ for a sufficient amount of time allowing basic forms of life to emerge and develop. In order to adhere to this criterion, planetary orbital stability is required. There is a significant body of literature devoted to this topic, including studies of binary and multi-planetary systems, which often also consider aspects of stellar evolution (e.g., Jones et al. 2001; Noble et al. 2002; Menou \& Tabachnik 2003; Sándor et al. 2007; Takeda et al. 2008; Dvorak et al. 2010; Haghighipour et al. 2010; Kopparapu \& Barnes 2010).

Early studies of planetary orbital stability pertaining to planets in both S-type and P-type orbits demonstrated that planets can exist in systems of binary stars for 3000 binary periods Dvorak (1984, 1986). Although these investigations considered relatively short integration times, Dvorak determined upper and lower bounds of planetary orbital stability considering the orbital elements, semimajor axis and eccentricity, of the proposed binary stars. Since this pioneering work, many additional studies have been performed. The foremost investigation extended the original study by a factor of 10 in integration times and an extended range of orbital elements (Holman \& Wiegert 1999). In addition, the nature of the bounding formula was derived and discussed using a more statistical framework. Holman \& Wiegert developed fitting formulae for both S-type and P-type planets in binary systems given as

$$
\frac{a_{\mathrm{cr}}}{a}=0.464-0.38 \mu+\mathcal{F}_{\mathrm{S}}\left(\mu, e_{\mathrm{b}}\right)
$$


and

$$
\frac{a_{\mathrm{cr}}}{a}=1.60+4.12 \mu+\mathcal{F}_{\mathrm{P}}\left(\mu, e_{\mathrm{b}}\right),
$$

respectively.

These equations give the critical semimajor axis $a_{\mathrm{cr}}$ in units of the semimajor axis $a$ in case of S-type and P-type orbits. For an S-type orbit, the ratio $a_{\text {cr }} / a$, see Eq. (46), conveys the upper limit of planetary orbital stability, whereas for a P-type orbit, the ratio $a_{\mathrm{cr}} / a$, see Eq. (47), conveys the lower limit of planetary orbital stability. Moreover, $\mu$ denotes the stellar mass ratio given as $\mu=M_{2} /\left(M_{1}+M_{2}\right)$, where $M_{1}$ and $M_{2}$ constitute the two masses of the binary components with $M_{2} \leq M_{1}$. Equations (46) and (47) also contain the parameter functions $\mathcal{F}_{\mathrm{S}}\left(\mu, e_{\mathrm{b}}\right)$ and $\mathcal{F}_{\mathrm{P}}\left(\mu, e_{\mathrm{b}}\right)$, which depend on the aforementioned mass ratio $\mu$ and the eccentricity of the stellar binary, $e_{\mathrm{b}}$. Considering that this paper is solely aimed at stellar binaries in circular orbits (i.e., $e_{\mathrm{b}}=0$ ), it is found that $\mathcal{F}_{\mathrm{S}}=\mathcal{F}_{\mathrm{P}}=0$.

Planetary orbital stability has been investigated by many authors using chaos indicators, such as the maximal Lyapunov exponents (MLE), fast Lyapunov indicator (FLI), and the mean exponential growth factor of nearby orbits (MEGNO), to name those commonly used; see, e.g., Satyal et al. (2013) for details, recent applications, and references. These methods have also been used to characterize the transition from stable to unstable orbits within the framework of the circular and elliptical 3-body problems; see, e.g., Cuntz et al. (2007), Eberle et al. (2008), and Szenkovits \& Makó (2008) for details.

Previously, Musielak et al. (2005) studied the stability of both S-type and P-type orbits in stellar binary systems, and deduced orbital stability limits for planets. These limits were found to depend on the mass ratio between the stellar components and the distance ratio between planetary and binary semimajor axes. This topic was revisited by Eberle et al. (2008), who used the concept of Jacobi's integral and Jacobi's constant to deduce stringent criteria for the stability of planetary orbits in binary systems for the special case of the coplanar circular restricted three-body problem. Recently the planetary orbital stability was studied through the perspective of a chaos indicator, the MLE by, e.g., Quarles et al. (2011). From the use of a chaos indicator a cutoff value for the maximum Lyapunov exponent was determined as an additional stability criterion for S-type planets in the circular restricted 3-body problem. 


\section{Case Studies}

\subsection{S-Type Habitability in Binary Systems}

Next we investigate S-type habitability for selected binary systems, including systems of equal and non-equal masses (see Table 5). Our main intent is to demonstrate the functionality of the method-as-proposed 5 , an extensive parameter study will be given in Sect. 6 . Figure 5 allows comparative insight into S-type habitability for selected binary systems, i.e., systems with masses of $M_{1}=M_{2}=1.0 M_{\odot}$ and $M_{1}=1.5 M_{\odot}, M_{2}=1.0 M_{\odot}$; the binary separation distances are chosen as $10 \mathrm{AU}$ and $20 \mathrm{AU}$. For single stars of $1.0 M_{\odot}$, the radiative $\mathrm{CHZ}$ extends from 1.049 to $1.498 \mathrm{AU}$, and the radiative GHZ extends from 0.927 to 1.831 AU; these values are slightly higher than those for G2 V stars given in Table 3 owing to a minuscule difference in mass (i.e., 0.99 versus $1.0 M_{\odot}$ ).

For an equal-mass binary system of $1.0 M_{\odot}$ with a separation distance $2 a$ of $10 \mathrm{AU}$, the radiative $\mathrm{CHZ}$ and $\mathrm{GHZ}$ extend from 1.056 to $1.511 \mathrm{AU}$, and from 0.932 to $1.853 \mathrm{AU}$, respectively, for each component. Furthermore, the outer limit of the radiative EHZ is altered from 2.64 to $2.70 \mathrm{AU}$. However, there is now an upper orbital stability limit of 1.37 AU imposed on each star. Consequently, significant portions of the radiative CHZ and GHZ are unavailable as circumstellar habitable regions. If the second star is placed at a distance of $20 \mathrm{AU}$, the alteration of the radiative $\mathrm{CHZ}$ and GHZ relative to single stars is very minor. Specifically, for binary separations of $10 \mathrm{AU}$ and $20 \mathrm{AU}$, the sizes of the radiative GHZ increase by $1.9 \%$ and $0.6 \%$ relative to the case of single stars. Moreover, for the system with a separation distance of $20 \mathrm{AU}$, the imposed orbital stability limit is found at $2.74 \mathrm{AU}$; consequently, the full extents of the radiative $\mathrm{CHZ}$, GHZ, and EHZ are now available for planetary habitability.

Figure 5 also shows results for the pairs $M_{1}=1.5 M_{\odot}$ and $M_{2}=1.0 M_{\odot}$. In case of a single $1.5 M_{\odot}$ mass star, the radiative $\mathrm{CHZ}$ and $\mathrm{GHZ}$ extend from 1.88 to $2.49 \mathrm{AU}$, and from 1.65 to $3.11 \mathrm{AU}$, respectively, whereas the radiative EHZ extends up to $4.61 \mathrm{AU}$. In this type of system, a secondary star of $1.0 M_{\odot}$ placed at a separation distance of $10 \mathrm{AU}$ again modifies the extents of the radiative $\mathrm{CHZ}$ and GHZ, which now extend from 1.89 to 2.49 AU and from 1.66 to $3.14 \mathrm{AU}$, respectively; however, the planetary orbital stability limit now occrs at $1.56 \mathrm{AU}$. Therefore, the entire domains of the radiative CHZ, GHZ, and EHZ of the primary are unavailable as circumstellar habitable regions. If the secondary star is placed

\footnotetext{
${ }^{5}$ The method has also successfully been used for determining the radiative habitable zone of Kepler-16, a binary system with $M_{1}=0.69 M_{\odot}$ and $M_{2}=0.20 M_{\odot}$, and a Saturnian planet in a P-type orbit; see Quarles et al. (2012) for a detailed study of the system's habitability.
} 
at a separation distance of $20 \mathrm{AU}$, the radiative $\mathrm{CHZ}, \mathrm{GHZ}$, and EHZ of the primary star are again similar to those of a $1.5 M_{\odot}$ mass star. However, the orbital stability limit is now found at a distance of $3.12 \mathrm{AU}$ from the primary; therefore, the entire supplement of the radiative EHZ, given by the bracket $\left(s_{6}-s_{5}\right)$ is now considered habitable.

In summary, for potentially habitable S-type binaries, owing to the implied requirement of the relatively large separations of the stellar components, the effect of the stellar secondary on the extents of the RHZs is often minor, i.e., about a few percent or less, with the biggest impact occurring in F-type systems. For most systems, the secondary's main influence on circumstellar habitability thus consists in limiting planetary orbital stability rather than offering significant augmentations of the RHZs, a feature most pronounced in close binaries.

\subsection{P-Type Habitability in Binary Systems}

\subsubsection{Case Studies}

Various sets of models have been pursued to examine P-type habitability (see Fig. 6). As examples we considered systems with masses of $M_{1}=M_{2}=1.0 M_{\odot}$ and $M_{1}=1.5 M_{\odot}$ and $M_{2}=0.5 M_{\odot} ;$ additionally, we also focused on models of $M_{1}=1.25 M_{\odot}$ and $M_{2}=0.75 M_{\odot}$ (see Tables 5 to 8 for details). The separation distances $2 a$ were chosen as $0.5,1.0$, and 2.0 AU, respectively. Our approach consists again of two steps. First, we explore the existence and extent of the radiative CHZs, GHZs, and EHZs. Subsequently, we consider the additional constraint of planetary orbital stability, which in the case of P-type orbits constitutes a lower limit (see Sect. 4). Our results can be summarized as follows.

For systems with masses of $M_{1}=M_{2}=1.0 M_{\odot}$, the following behavior is found. For separation distances $2 a$ of $0.5 \mathrm{AU}$, the inner limit (i.e., RHL; see Sect. 3.4) of the radiative $\mathrm{CHZ}$ varies between 1.46 and $1.54 \mathrm{AU}$ as function of polar angle $\varphi$ with $1.54 \mathrm{AU}$ to be considered as acceptable inner limit; see Eq. (39a). Furthermore, the outer limit of the radiative $\mathrm{CHZ}$ varies between 2.10 and $2.16 \mathrm{AU}$ with $2.10 \mathrm{AU}$ as acceptable outer limit; see Eq. (39b). In consideration of the orbital stability limit at $0.92 \mathrm{AU}$ (see Eq. 45), constituting an inner limit of orbital stability, the entire extent of the radiative CHZ is available as a circumbinary habitable region. The acceptable inner limit of the radiative GHZ is given as $1.38 \mathrm{AU}$, whereas the acceptable outer limit occurs at $2.58 \mathrm{AU}$; hence, the entire radiative GHZ is again identified as habitable.

For separation distances of 1.0 AU, the orbital stability limit is given at $1.83 \mathrm{AU}$, which falls inside the domain of the radiative $\mathrm{CHZ}$ ranging from 1.69 to $2.06 \mathrm{AU}$; therefore, only about half of the radiative CHZ is available for circumbinary habitability, whereas the other 
half is not. Since the radiative CHZ is fully embedded into the radiative GHZ, only a fraction of the radiative GHZ offers circumbinary habitability. However, the full extent of the supplementary radiative EHZ, given by the bracket $\left(s_{6}-s_{5}\right)$, with an acceptable outer limit of $3.83 \mathrm{AU}$, offers habitability. We also considered models with binary separations of $2.0 \mathrm{AU}$. In this case, the orbital stability limit is found at $3.66 \mathrm{AU}$. Therefore, both the radiative $\mathrm{CHZ}$ and $\mathrm{GHZ}$ are unavailable for providing habitability; the latter has an outer limit that varies between 2.39 and 3.05 AU with 2.39 AU as acceptable limit. The outer limit of the radiative EHZ varies between 3.60 and 4.09 AU with 3.60 AU to be ruled acceptable as the conservatively selected (i.e., inner) limit of the radiative EHZ. Hence, the entire radiative EHZ is also not considered available for providing circumbinary habitability.

Most significantly, we also pursued case studies for systems of unequal distributions of mass, and by implication of unequal distributions of luminosity as, for example, the system $M_{1}=1.5 M_{\odot}$ and $M_{2}=0.5 M_{\odot}$. According to the mass-luminosity relationship for main-sequence stars, it is found that a $1.5 M_{\odot}$ star possesses a luminosity $L_{*}$ about 3.5 times higher than a $1.0 M_{\odot}$ star; a similar factor of difference exists for the recast stellar luminosity $L_{i \ell}^{\prime}$ (see Tables 4 and 5$)$. Thus, the combined luminosity of the $\left(1.5 M_{\odot}, 0.5 M_{\odot}\right)$ system is considerably higher than the combined luminosity of the $\left(1.0 M_{\odot}, 1.0 M_{\odot}\right)$ system, as expected. On the other hand, following the work by Dvorak (1986) and Holman \& Wiegert (1999), an unequal distribution of stellar mass, i.e., a smaller value of $\mu$ (see Sect. 4), entails a smaller orbital stability limit. Since it constitutes a lower limit, i.e., positioned more closely to the stellar system, it offers larger "windows of opportunity" for planets in the RHZs (if existing) to be orbitally stable.

Results for separation distances $2 a$ of 0.5 , 1.0, and $2.0 \mathrm{AU}$ are given in Fig. 6. For a binary separation of $0.5 \mathrm{AU}$, it is found that both the radiative $\mathrm{CHZ}$ and $\mathrm{GHZ}$ exist, and habitability in these domains is fully permitted according to the planetary orbital stability constraint, although the width of the $\mathrm{CHZ}$ is relatively small. The CHZ extents from 2.14 to 2.28 AU, whereas the GHZ extents from 1.91 to $2.90 \mathrm{AU}$; the orbital stability limit is given at $0.66 \mathrm{AU}$. In this type of system, there are extreme variations for the inner and outer limits of both the radiative $\mathrm{CHZ}$ and GHZ. For example, the inner RHL for the CHZ varies between 1.65 and 2.14 AU, whereas its outer RHL varies between 2.28 and 2.76 AU as function of polar angle $\varphi$. There is also a considerably large domain of the supplementary portion of the radiative EHZ, which has an outer limit that varies between 4.40 and 4.89 AU. Detailed depictions of the variations of the inner and outer limits of the RHZs for the various systems are given in Fig. 7. This figure indicates relatively small bars of variations for equal-mass systems such as $M_{1}=M_{2}=1.0 M_{\odot}$ with small separation distances as, e.g., $2 a=0.5$ AU. However, large bars of variations are obtained for non-equal mass binaries or for equal-mass binaries with large separation distances as, e.g., $2 a=2.0$ AU. 
In systems with a binary separation of $1.0 \mathrm{AU}$, the radiative $\mathrm{CHZ}$ is nullified; note that the orbital stability limit in this system is given at $1.32 \mathrm{AU}$. The reason for the disallowance of the CHZ is that the RHL for $s_{4}$, which is at $2.38 \mathrm{AU}$, is located inside of the RHL for $s_{2}$, given as $2.05 \mathrm{AU}$. The same criterion (see Eq. 40) also leads to a relatively small width of the radiative GHZ, which extends between 2.16 and $2.66 \mathrm{AU}$. At a binary separation of $2.0 \mathrm{AU}$, the situation is even more drastic as both the radiative CHZ and GHZ are disallowed. The only type of circumbinary habitable region remaining is that provided by the relatively large supplementary portion of the radiative EHZ given by the bracket $\left(s_{6}-s_{5}\right)$. In this zone, habitable planets are expected to be possible as their existence would be consistent with the planetary orbital stability constraint.

\subsubsection{Additional Analyses}

Next we explore the existence of P-type RHZs, both for equal-mass and non-equal mass binaries, in a more systematic manner through the means of numerical experiments. Specifically, we pursue sets of model calculations with the binary separation distance $2 a$ considered as an independent variable; see Table 9 for results. The stellar masses are altered between $0.5 M_{\odot}$ and $1.5 M_{\odot}$ in increments of $0.25 M_{\odot}$ (see Table 5). Results are given for the pairings $\left(s_{2}, s_{4}\right)(\mathrm{CHZ}),\left(s_{1}, s_{5}\right)$ (GHZ), and $\left(s_{1}, s_{6}\right)$ (EHZ). Note that for equalmass binaries, it is sufficient to solve Eq. (45), whereas for general binary systems a more thorough assessment is needed to satisfy relation (40). This approach allows us to explore the maximum binary separation distances, which are upper limits for permitting RHZs for each case (i.e., combination of binary masses and choice of CHZ, GHZ, or EHZ).

Generally, it is found that for any binary system, the greatest permissible binary separation distance is attained for the EHZ, and furthermore that value-as-attained is greater for the GHZ than for the CHZ; these findings are as expected. For example, for the system $M_{1}=M_{2}=1.0 M_{\odot}$, the expiration distance for the radiative EHZ is given as $4.25 \mathrm{AU}$, whereas for the radiative GHZ and CHZ, the distances are given as 2.57 and $1.64 \mathrm{AU}$, respectively (see Table 9). As another example, the system $M_{1}=1.25 M_{\odot}$ and $M_{2}=0.75 M_{\odot}$, the expiration distances for the radiative EHZ, GHZ, and CHZ are given as 3.80, 1.93, and $0.96 \mathrm{AU}$, respectively.

It is also intriguing to compare results for stellar pairs as, e.g., $M_{1}=M_{2}=1.0 M_{\odot}$ to stellar pairs such as $M_{1}=1.5 M_{\odot}$ and $M_{2}=0.5 M_{\odot}$. For the system of $M_{1}=M_{2}=1.0 M_{\odot}$, it is found that the radiative CHZ and GHZ are nullified - as defined by the limit of validity of Eq. (45) - at binary separation distances of 1.64 and 2.57 AU, respectively (see Table 9). At those binary separations, the distances of the vanishing RHZ-CHZ and RHZ-GHZ (as 
measured from the geometrical center of the system; see Fig. 1) are given as 1.95 and 2.25 AU, respectively. In comparison, the limits of planetary orbital stability (to be interpreted as lower limits) are identified as 3.00 and $4.71 \mathrm{AU}$, respectively. Thus, we conclude that for equal-mass binary systems such as $M_{1}=M_{2}=1.0 M_{\odot}$, habitability for widely spaced binaries is lost due to the lack of orbital stability already at binary separations where the circumbinary CHZ-RHZs and GHZ-RHZs are still in place.

The same type of study has been pursued for systems with highly unequal intrabinary distributions of masses and, by implication, stellar luminosities as, e.g., $M_{1}=1.5 M_{\odot}$ and $M_{2}=0.5 M_{\odot}$. In this case it is found that the radiative CHZ and GHZ vanish at binary separation distances $2 a$ of 0.65 and 1.55 AU, respectively. Furthermore, the distances of the vanishing radiative $\mathrm{CHZ}$ and GHZ (as measured from the system center, see Fig. 1) are given as 2.21 and 2.43 AU, respectively (see Table 9). The respective limits of planetary orbital stability are identified as 0.85 and 2.04 AU. Thus, for this type of system it is found that habitability is lost due to the vanishing RHZs, even though circumstellar habitability would still be permitted according to the planetary orbital stability criterion. The fact that circumbinary habitability is lost already for systems of relatively small binary separations is a consequence of the extreme radiative imbalance caused by the highly unequal distribution of stellar luminosities, which determine the circumbinary RHLs.

Radiative imbalance within binary systems may cause the RHL for $s_{\ell, \text { out }}$ to be partially or completely located inside of the RHL for $s_{\ell \text {,in }}$; see Sect. 3.4. In fact, when the pairs $M_{1}=M_{2}=1.0 M_{\odot}$ and $M_{1}=1.5 M_{\odot}$ and $M_{2}=0.5 M_{\odot}$ are compared to one another, it is found that although the unequal-mass binary system has almost twice the combined stellar luminosity of the equal-mass binary system (i.e., 3.85 versus $2.0 L_{\odot}$ ), it still possesses much narrower CHZ, GHZ, and EHZ RHZs. In fact, it is found that the condition expressed as Eq. (40) is most readily met in cases of equal-mass binary systems of relatively small separation distances and mostly violated in systems of relatively large separation distances and/or unequal distributions of masses and, by implication, luminosities. Various examples have been depicted in Fig. 7; see discussion in Sect. 5.2.1.

In summary, although an unequal distribution of stellar masses within binary systems is identified as advantageous for facilitating planetary orbital stability, in consideration of that lower stability limits for P-type orbits occur for smaller mass ratios $\mu$ (see Sect. 4), the situation for the existence of the RHZs is much less ideal, even for systems where the stellar primary is highly luminous owing to the behavior of the RHLs. In this regard, the radiative CHZ is in most jeopardy as $\left(s_{4}-s_{2}\right)$ constitutes the smallest bracket among the various kinds of HZs (see Table 2). More fortunate scenarios are expected to occur for the radiative GHZ and EHZ, with the brackets given as $\left(s_{5}-s_{1}\right)$ and $\left(s_{6}-s_{1}\right)$, respectively; they are 
characterized by considerably larger widths, especially in case of equal-mass systems of stars with relatively high luminosities.

\section{Proposed Habitability Classification: Habitability Types S, P, ST, and PT}

Another aspect of this study is to provide an appropriate classification of habitability applicable to general binary systems. Previously, Dvorak (1982) introduced the terminology of S-type and P-type orbits for system planets, which is now widely used by the orbital stability, planetary, and the astrobiology science communities. Evidently, besides the assessment of orbital stability behaviors, these terms are also appropriate for classifying binary system RHZs, if existing. However, following previous investigations (e.g., Takeda et al. 2008; Dvorak et al. 2010; Haghighipour et al. 2010; Kopparapu \& Barnes 2010; Eggl et al. 2012), as well as the results of the present work, the spatial domain of S-type and P-type habitability depicted by the RHZs is often adversely affected, and in some cases even nullified, by the requirement that system planets must be orbitally stable. Thus, if the available extent of the S-type and P-type RHZs for the manifestation of habitability is truncated owing to the additional constraint of planetary orbital stability, these zones shall be referred to as ST-type and PT-type, respectively, in the following.

Detailed results are given in Table 10, which provides an extensive summary of P, PT, ST, and S-type habitability for both equal-mass and non-equal mass binary systems. The stellar masses are varied between $0.5 M_{\odot}$ and $1.5 M_{\odot}$ in increments of $0.25 M_{\odot}$ amounting to a total of 15 combinations. Table 10 features the results for the pairings $\left(s_{2}, s_{4}\right)(\mathrm{CHZ})$, $\left(s_{1}, s_{5}\right)(\mathrm{GHZ})$, and $\left(s_{1}, s_{6}\right)(\mathrm{EHZ})$. In principle, it is found that - with the secondary taken as fixed - the higher the mass and, by implication, the luminosity of the stellar primary, the larger values are obtained for P, PT, ST, and S-type habitability. Additionally, larger values for the limits of P, PT, ST, and S-type habitability are obtained regarding the GHZ relative to the CHZ, as expected. The largest values are obtained for PT and S-type habitability for the EHZ; in this regard, there is no change for $\mathrm{P}$ and ST-type habitability relative to the GHZ since both types of HZs are based on the same inner bracket value of $s_{1}$ (see above).

The results of Table 10 are in line with the previously discussed findings about highly unequal intrabinary distributions of masses and, by implication, stellar luminosities as, e.g., $M_{1}=1.25 M_{\odot}$ and $M_{2}=0.75 M_{\odot}$ or $M_{1}=1.5 M_{\odot}$ and $M_{2}=0.5 M_{\odot}$ compared to the case of $M_{1}=M_{2}=1.0 M_{\odot}$. For systems of highly unequal mass distributions, the domains of Ptype and PT-type habitability are typically relatively small as the RHL for $s_{\ell, \text { out }}$ crosses the RHL for $s_{\ell, \text { in }}$ in relatively close proximity to the primary, such allowing only small distance ranges to exhibit $\mathrm{P} / \mathrm{PT}$-type habitability. It is also found that in seven cases for the $\mathrm{CHZ}$, 
as well as two cases for the GHZ, the RHZs expire prior to the truncation of habitability due to the planetary orbital stability requirement. In those cases, only P-type habitability exists; no PT-type habitability is found as the orbital stability constraint bears no relevance.

Figure 8 and 9 show various combinations of equal-mass and nonequal-mass binary systems; they all show numerous similarities, though the spatial scales are noticeably different as they are defined through the stellar luminosities. If equal-mass binary systems are considered, taking $M_{1}=M_{2}=1.0 M_{\odot}$ and $M_{1}=M_{2}=0.5 M_{\odot}$ as examples, the extent of both $\mathrm{P}$ and PT-type habitability increase with increasing stellar mass or luminosity. The distances for P and PT habitability are found to almost coincide indicating that the orbital stability constraint affects the inner and outer limit of P-type habitability in about the same manner. Furthermore, the inner and outer limits of both S and ST habitability are shifted to larger distances from each stellar component for stars of higher luminosity, as expected. Moreover, for stars of higher luminosity, there is a larger spatial domain where S-type habitability is truncated due to the additional constraint of planetary orbital stability. For equal-mass systems of $1.0 M_{\odot}$, ST and S-type habitability is identified at distances of 6.85 and $13.46 \mathrm{AU}$, whereas for $0.5 M_{\odot}, \mathrm{ST}$ and S-type habitability is identified at 1.38 and $3.00 \mathrm{AU}$.

Figure 9 depicts two selected cases of nonequal-mass binary systems. In both cases the stellar primary is chosen as $1.0 M_{\odot}$, whereas the stellar secondary is chosen as $0.75 M_{\odot}$ and $0.5 M_{\odot}$, respectively; the corresponding stellar luminosities of the secondaries are 0.357 and $0.045 L_{\odot}$, respectively (see Table 5 ). A reduced luminosity of the secondary binary component adversely affects the extent of the RHZ, as expected. Interestingly, a reduction of mass for the stellar secondary has a nontrivial impact on the orbital stability domains, which considerably depend on the mass ratio $\mu$ (see Eqs. 46 and 47). If $\mu$ is reduced, the permissible stability domain for P-type orbits is increased, whereas the permissible stability domain for S-type orbits is decreased. Thus, the assessment of S, P, ST, and PT habitability for nonequal-mass binaries requires a detailed computational analysis. In the example of Fig. 9, in regard to $M_{2}=0.75 M_{\odot}, \mathrm{S}, \mathrm{P}, \mathrm{ST}$, and PT habitability occurs at distances of 0.82 , 1.16, 6.18, and 12.19 AU, respectively, and for $M_{2}=0.5 M_{\odot}, \mathrm{S}, \mathrm{P}, \mathrm{ST}$, and PT habitability occurs at distances of $0.94,1.01,5.50$, and $10.86 \mathrm{AU}$, respectively. The respective differences are notable, but not drastic; more pronounced differences occur for systems of more luminous stars (see Table 10). Note that both Figs. 8 and 9 refer to habitability assessments pertaining to the GHZ (see Table 2). They are given to exemplarily showcase the structure, extent and location of the S-type and P-type RHZs as well as the relevance of the orbital stability limits for both S-type and P-type habitability, thus allowing us to uniquely identify the spatial domains of S, P, ST, and PT-type habitability for each system. 
Computationally, the occurrence of P, PT, ST, and S-type habitability can be identified as follows. For sufficiently small binary separations $2 a$, it is found that both the inner and outer limit of the P-type RHZ are located beyond of the P-type orbital stability limit (see Eq. 47), which constitutes a lower limit of planetary orbital stability (see Sect. 4). If the distance of binary separation is increased, the inner and outer limits of the P-type RHZ decrease, whereas the P-type orbital stability limit increases. Thus, starting at a certain value of $2 a$, only a fraction of the width of the P-type RHZ will be available for providing habitability; in this case, PT-type habitability is attained. If the binary separation is further increased, the entire width of the P-type RHZ will be unavailable for providing habitability because habitability would be incompatible with the orbital stability constraint. Eventually, the P-type RHZ expires; see also information provided in Table 9.

For mid-sized values of the binary separation distance, the S-type RHZ is encountered to exist, but it is unable to provide habitability because of the S-type orbital stability limit (see Eq. 46), which constitutes an upper limit of planetary orbital stability (see Sect. 4). The S-type RHZs continue to exist further out; note that the inner and outer limits of the S-type RHZs essential continue to run parallel as function of the binary separation distance for most systems. If the binary separation distance $2 a$ is further increased, again as part of our numerical experiment, the S-type orbital stability will increase and will cross the inner limit of the S-type RHZ; in this case, ST-type habitability is encountered as some, but not all of the width of the S-type RHZ is available for providing habitability. Eventually, for sufficiently large binary separations, the S-type orbital stability limit also crosses the outer limit of the S-type RHZ. In this case, the full width of the S-type RHZ is available for facilitating habitability, consistent with the definition of S-type habitability.

Another application is displayed in Fig. 10. It shows for a given spectral type of equalstar binaries the stellar separation distances $2 a$ for which CHZs, GHZs, and EHZs are able to exist. The CHZs, GHZs, and EHZs can be either S or ST-type, on one hand, or P or PTtype, on the other hand, to qualify for depiction. The results are given as function of stellar spectral type, for stars between spectral type F0 to M0. The figure shows that P/PT-type habitable regions are able to exist for a relatively large range of separation distances in case of relatively luminous stars (i.e., spectral type F), but only for a relatively small range of separation distances for lesser luminous stars (i.e., spectral types K and M). Regarding S/STtype habitable regions the situation is reversed. Figure 10 also indicates a notable domain of binary separations where no habitable regions are found owing to the lack of RHZs, the lack of planetary orbital stability, or both. Moreover, no domain of binary separation distances is identified where S/ST-type and P/PT-type habitable regions overlap. 


\section{Summary and Conclusions}

In this study we present a new method about a comprehensive assessment of S-type and P-type habitability in stellar binary systems. P-type orbits occur when the planet orbits both binary components, whereas in case of S-type orbits the planet orbits only one of the binary components with the second component considered a perturbator. An important characteristic of the new method is that it combines the orbital stability constraint for a system planet with the necessity that a habitable region given by the stellar radiative energy fluxes ("radiative habitable zone") must exist. The requirement to combine these two properties has also been recognized in previous studies (e.g., Takeda et al. 2008, Kopparapu \& Barnes 2010; Eggl et al. 2012; Kane \& Hinkel 2013).

Another element of the present study is to introduce a habitability classification regarding stellar binary systems, consisting of habitability types S, P, ST, and PT. This type of classification also considers whether or not S-type and P-type radiative habitable zones are reduced in size due to the additional constraint of planetary orbital stability. In summary, five different cases were identified, which are: S-type and P-type habitability provided by the full extent of the RHZ; habitability, where the RHZ is truncated by the additional constraint of planetary orbital stability (labelled as ST and PT-type, respectively); and cases of no habitability at all. This classification scheme can be applied to both equal-mass and non-equal mass binary systems, as well as to systems with binaries in elliptical orbits, which will be the focus of the forthcoming Paper II of this series. As part of the current study a significant array of results are given for a notable range of main-sequence stars, which are of both observational and theoretical interest.

A key aspect of the proposed method is the introduction of a combined algebraic formalism for the assessment of both S-type and P-type habitability; in particular, mathematical criteria are presented allowing to determine for which systems S-type and P-type RHZs are realized. In this regard, a priori choices about the presence of S-type and P-type RHZs are neither necessary nor possible as the existence of S-type as well as P-type RHZs is proliferated through well-defined mathematical conditions pertaining to the underlying fourth-order algebraic equation. The coefficients of the polynomial are given by the binary separation distance $(2 a)$, the solar system-based parameter for the limit of habitability $\left(s_{\ell}\right)$, and the modified values for the luminosities $\left(L_{1 \ell}^{\prime}, L_{2 \ell}^{\prime}\right)$ of the stellar binary components, referred to as recast stellar luminosities. Regarding the binary system habitable zone, we consider conservative, general and extended zones of habitability, noting that their inner and outer limits are informed by previous solar system investigations (e.g., Kasting et al. 1993; Underwood et al. 2003; Selsis et al. 2007). 
In our segment of applications, we examined the existence of habitable S-type orbits for selected examples. We found that regarding the RHZs, owing to the typically relatively large separation of the stellar components, the effect of the stellar secondary on the extents of the RHZs is usually very minor. The secondary's main influence on circumstellar habitability consists in imposing restrictions regarding planetary orbital stability implemented as an upper stability limit around each stellar component, which often truncates or nullifies S-type planetary habitability. In the framework of our study, we specifically considered the radiative EHZ, which is most outwardly extented (i.e., up to $2.4 \mathrm{AU}$ in case of the Sun). It was found that this kind of zone is most affected by the limitation of planetary orbital stability as it is located closest to the secondary stellar component.

Furthermore, we also examined the existence of habitable P-type orbits. In this case, relatively complicated scenarios emerge. In general, it was found that the best prospects for circumbinary habitability emerge for (1) systems with stellar components of relatively high luminosities (no surprise here!), (2) systems where the stellar luminosities are relatively similar (for main-sequence stars, as implied by their stellar masses), and (3) systems of relatively small binary separations. If conditions (2) or (3) are not met, it may occur that the outer RHL is located inside of the inner RHL, thus nullifying the RHZ irrespectively of planetary orbital stability considerations. On the other hand, an unequal intrabinary distribution of masses entails a lower limit of planetary orbital stability (i.e., positioned closer to the binary system) thus implying an enhanced opportunity for circumbinary habitability. However, this aspect is of lesser significance for most systems compared to the restrictions for the RHZs due to the imbalance given by the stellar luminosities.

Various applications in this study concern stars of masses between 0.75 and $1.5 M_{\odot}$. This approach is motivated to unequivocally demonstrate the effects of stellar binarity on the extent and structure of circumstellar habitability, which is most pronounced for massive, i.e., highly luminous stars. Nonetheless, most stars in binaries are expected to be low-mass stars, i.e., stars of spectral types $\mathrm{K}$ and $\mathrm{M}$, owing to the skewness of the Galactic initial mass function (e.g., Kroupa 2001, 2002; Chabrier 2003). For example, we compared pairs of systems given by $\left(1.0 M_{\odot}, 1.0 M_{\odot}\right)$ and $\left(1.5 M_{\odot}, 0.5 M_{\odot}\right)$. Obviously, the overall luminosity is by far greatest in the $\left(1.5 M_{\odot}, 0.5 M_{\odot}\right)$ system following the mass-luminosity relationship, i.e., $L_{*} \propto M_{*}^{4}$ (e.g., $\left.\operatorname{Reid} 1987\right)$. However, this system is found to be the highly unfavorable for the facilitation of circumbinary habitability. Particularly, it is found that the P-type GHZ in the $\left(1.0 M_{\odot}, 1.0 M_{\odot}\right)$ system extends to $0.91 \mathrm{AU}$, whereas it extends only to $0.65 \mathrm{AU}$ in the $\left(1.5 M_{\odot}, 0.5 M_{\odot}\right)$ system. Furthermore, smaller spatial extents are identified for P-type CHZs, as this type of HZ is in highest jeopardy owing to the relative small $\left(s_{4}-s_{2}\right)$ bracket compared to the $\left(s_{5}-s_{1}\right)$ bracket for GHZs (see Table 2). In fact, a considerable number of systems do not offer CHZs at all, which again is a consequence of the radiative imbalance 
in those systems. Also, the nullification of CHZs in binary systems is most likely to occur in systems of relatively large separation distance. In contrast, the best opportunities for facilitating circumbinary habitability is given in the context of EHZs, as expected.

Future work will deal with a significant augmentation of our method to other systems, including systems with binary components in elliptical orbits (see Paper II). This will allow us to compare applications of our method, including results for individual systems, to other findings in the literature. We also expect our method to be applicable to general binary systems with main-sequence stars as well as to systems containing evolved stars; this latter effort is motivated by observational evidence and supporting theoretical efforts indicating that planets are also able to exist around stars that have left the main-sequence (e.g., Sato et al. 2003; Ramm et al. 2009; Eberle \& Cuntz 2010; Doyle et al. 2011; Sato et al. 2013). Particularly, it is highly desirable to augment our method to systems of higher order, as motivated by the steady progress in theory as well as ongoing and future observational discoveries of exosolar planetary systems.

This work has been supported in part by the SETI institute. The author acknowledges comments by B. Quarles and Z. E. Musielak as well as assistance with computer graphics by S. Sato, S. Satyal, and M. Sosebee. The paper also benefited from detailed comments by an anonymous referee. This study made use of the software applications Fortran ${ }^{\circledR}$, MATHEMATICA $^{\circledR}$, and MATLAB ${ }^{\circledR}$. The author anticipates the development of a black box code, called BinHab, to be hosted at The University of Texas at Arlington, which will allow the assessment of habitability in binary systems based on the developed method. 


\section{REFERENCES}

Abramowitz, M., \& Stegun, I. A. 1972, Handbook of Mathematical Functions with Formulas, Graphs, and Mathematical Tables, 9th printing (New York: Dover)

Asghari, N., Broeg, C., Carone, L., et al., 2004, A\&A, 426, 353

Beyer, W. H. 1987, Handbook of Mathematical Sciences, 6th edn. (Boca Raton: CRC Press)

Bonavita, M., \& Desidera, S. 2007, A\&A, 468, 721

Bronshtein, I. N., \& Semendyayev, K. A. 1997, Handbook of Mathematics, 3rd edn. (New York: Springer)

Castelli, F., \& Kurucz, R. L. 2004, in IAU Symp. 210, Modelling of Stellar Atmospheres ed. N. E. Piskunov, W. W. Weiss, \& D. F. Gray (San Francisco: ASP), CD-ROM Poster 20; arXiv:astro-ph/0405087

Chabrier, G. 2003, PASP, 115, 763

Cuntz, M., \& Yeager, K. E. 2009, ApJ, 697, L86

Cuntz, M., Eberle, J., \& Musielak, Z. E. 2007, ApJ, 669, L105

Cuntz, M., von Bloh, W., Schröder, K.-P., Bounama, C., \& Franck, S. 2012, Int. J. Astrobiol., 11,15

Doyle, L. R., Carter, J. A., Fabrycky, D. C., et al. 2011, Science, 333, 1602

Duquennoy, A., \& Mayor, M. 1991, A\&A, 248, 485

Dvorak, R. 1982, Österreichische Akademie der Wissenschaften, MathematischNaturwissenschaftliche Klasse, Sitzungsberichte Abt. 2, 191 (10), 423

Dvorak, R. 1984, Celest. Mech. Dyn. Astron., 34, 369

Dvorak, R. 1986, A\&A, 167, 379

Dvorak, R., Pilat-Lohinger, E., Bois, E., et al. 2010, Astrobiology, 10, 33

Eberle, J., \& Cuntz, M. 2010, ApJ, 721, L168

Eberle, J., Cuntz, M., \& Musielak, Z. E. 2008, A\&A, 489, 1329

Eggenberger, A., Udry, S., \& Mayor, M. 2004, A\&A, 417, 353 
Eggenberger, A., Udry, S., Chauvin, G., Beuzit, J.-L., Lagrange, A.-M., Ségransan, D., \& Mayor, M. 2007, A\&A, 474, 273

Eggl, S., Pilat-Lohinger, E., Georgakarakos, N., Gyergyovits, M., \& Funk, B. 2012, ApJ, 752, A74

Fatuzzo, M., Adams, F. C., Gauvin, R., \& Proszkow, E. M. 2006, PASP, 118, 1510

Forget, F. 2013, Int. J. Astrobiol., 12, 177

Forget, F., \& Pierrehumbert, R. T. 1997, Science, 278, 1273

Gray, D. F. 2005, The Observation and Analysis of Stellar Photospheres, 2nd edn. (Cambridge: Cambridge University Press)

Haghighipour, N., Dvorak, R., \& Pilat-Lohinger, E. 2010, in Planets in Binary Systems, ed. N. Haghighipour, Astrophysics and Space Science Library, Vol. 366 (New York: Springer Science + Business Media), p. 285

Halevy, I., Pierrehumbert, R. T., \& Schrag, D. P. 2009, J. Geophys. Res., 114, D18112

Holman, M. J., \& Wiegert, P. A. 1999, AJ, 117, 621

Horner, J., \& Jones, B. W. 2010, Int. J. Astrobiol., 9, 273

Jackson, J. D. 1999, Classical Electrodynamics, 3rd edn., John Wiley \& Sons, Inc., Hoboken, NJ

Jones, B. W., Sleep, P. N., \& Chambers, J. E. 2001, A\&A, 366, 254

Jones, B. W., \& Sleep, P. N. 2010, MNRAS, 407, 1259

Kaltenegger, L., Eiroa, C., Ribas, I., et al. 2010, Astrobiology, 10, 103

Kane, S. R., \& Hinkel, N. R. 2013, ApJ, 762, A7

Kasting, J. F., \& Catling, D. 2003, ARA\&A, 41, 429

Kasting, J. F., Whitmire, D. P., \& Reynolds, R. T. 1993, Icarus, 101, 108

Kopparapu, R. K., \& Barnes, R. 2010, ApJ, 716, 1336

Kroupa, P. 2001, MNRAS, 322, 231

Kroupa, P. 2002, Science, 295, 82 
Kurucz, R. L. 2005, Mem. S. A. It., 8, 14

Lada, C. J. 2006, ApJ, 640, L63

Lammer, H., Bredehöft, J. H., Coustenis, A., et al. 2009, A\&A Rev., 17, 181

Lammer, H., Selsis, F., Chassefière, E., et al. 2010, Astrobiology, 10, 45

Lucarini, V., Pascale, S., Boschi, R., Kirk, E., \& Iro, N. 2013, AN, 334, 576

Menou, K., \& Tabachnik, S. 2003, ApJ, 583, 473

Mischna, M. A., Kasting, J. F., Pavlov, A., \& Freedman, R. 2000, Icarus, 145, 546

Musielak, Z. E., Cuntz, M., Marshall, E. A., \& Stuit, T. D. 2005, A\&A, 434, 355

Noble, M., Musielak, Z. E., \& Cuntz, M. 2002, ApJ, 572, 1024

Orosz, J. A., Welsh, W. F., Carter, J. A., et al. 2012, Science, 337, 1511

Patience, J., White, R. J., Ghez, A. M., et al. 2002, ApJ, 581, 654

Perryman, M. 2011, The Exoplanet Handbook (Cambridge: Cambridge University Press)

Quarles, B., Eberle, J., Musielak, Z. E., \& Cuntz, M. 2011, A\&A, 533, A2

Quarles, B., Musielak, Z. E., \& Cuntz, M. 2012, ApJ, 750, A14

Quintana, E. V., \& Lissauer, J. J. 2010, in Planets in Binary Systems, ed. N. Haghighipour, Astrophysics and Space Science Library, Vol. 366 (New York: Springer Science + Business Media), p. 265

Raghavan, D., Henry, T. J., Mason, B. D., et al. 2006, ApJ, 646, 523

Raghavan, D., McAlister, H. A., Henry, T. J., et al. 2010, ApJS, 190, 1

Ramm, D. J., Pourbaix, D., Hearnshaw, J. B., \& Komonjinda, S., 2009, MNRAS, 394, 1695

Reid, N. 1987, MNRAS, 225, 873

Roell, T., Neuhäuser, R., Seifahrt, A., \& Mugrauer, M. 2012, A\&A, 542, A92

Sándor, Zs., Sűli, Á., Érdi, B., Pilat-Lohinger, E., Dvorak, R. 2007, MNRAS, 375, 1495

Scalo, J., Kaltenegger, L., Segura, A. G., et al. 2007, Astrobiology, 7, 85

Sato, B., Ando, H., Kambe, E., et al., 2003, ApJ, 597, L157 
Sato, B., Omiya, M., Harakawa, H., et al., 2013, PASJ, 65, 85

Satyal, S., Quarles, B., \& Hinse, T. C. 2013, MNRAS, 433, 2215

Selsis, F., Kasting, J. F., Levrard, B., Paillet, J., Ribas, I., \& Delfosse, X. 2007, A\&A, 476, 1373

Selsis, F., Kaltenegger, L., Paillet, J., et al. 2008, Physica Scripta, 130, 014032

Setiawan, J., Hatzes, A. P., von der Lühe, et al. 2003, A\&A, 398, L19

Slawson, R. W., Prša, A., Welsh, W. F., et al. 2011, AJ, 142, 160

Stix, M. 2004, The Sun: An Introduction, 2nd edn. (A\&A Library, Berlin: Springer)

Szenkovits, F., \& Makó, Z. 2008, Celest. Mech. Dyn. Astron., 101, 273

Takeda, G., Kita, R., \& Rasio, F. A. 2008, ApJ, 683, 1063

Tarter, J. C., Backus, P. R., Mancinelli, R. L., et al. 2007, Astrobiology, 7, 30

Trilling, D. E., Stansberry, J. A., Stapelfeldt, K. R., et al. 2007, ApJ, 658, 1289

Underwood, D. R., Jones, B. W., \& Sleep, P. N. 2003, Int. J. Astrobiol., 2, 289

Williams, D. M., \& Pollard, D. 2002, Int. J. Astrobiol., 1, 61

Zahnle, K., Arndt, N., Cockell, C., Halliday, A., Nisbet, E., Selsis, F., \& Sleep, N. H. 2007, Space Sci. Rev., 129, 35 

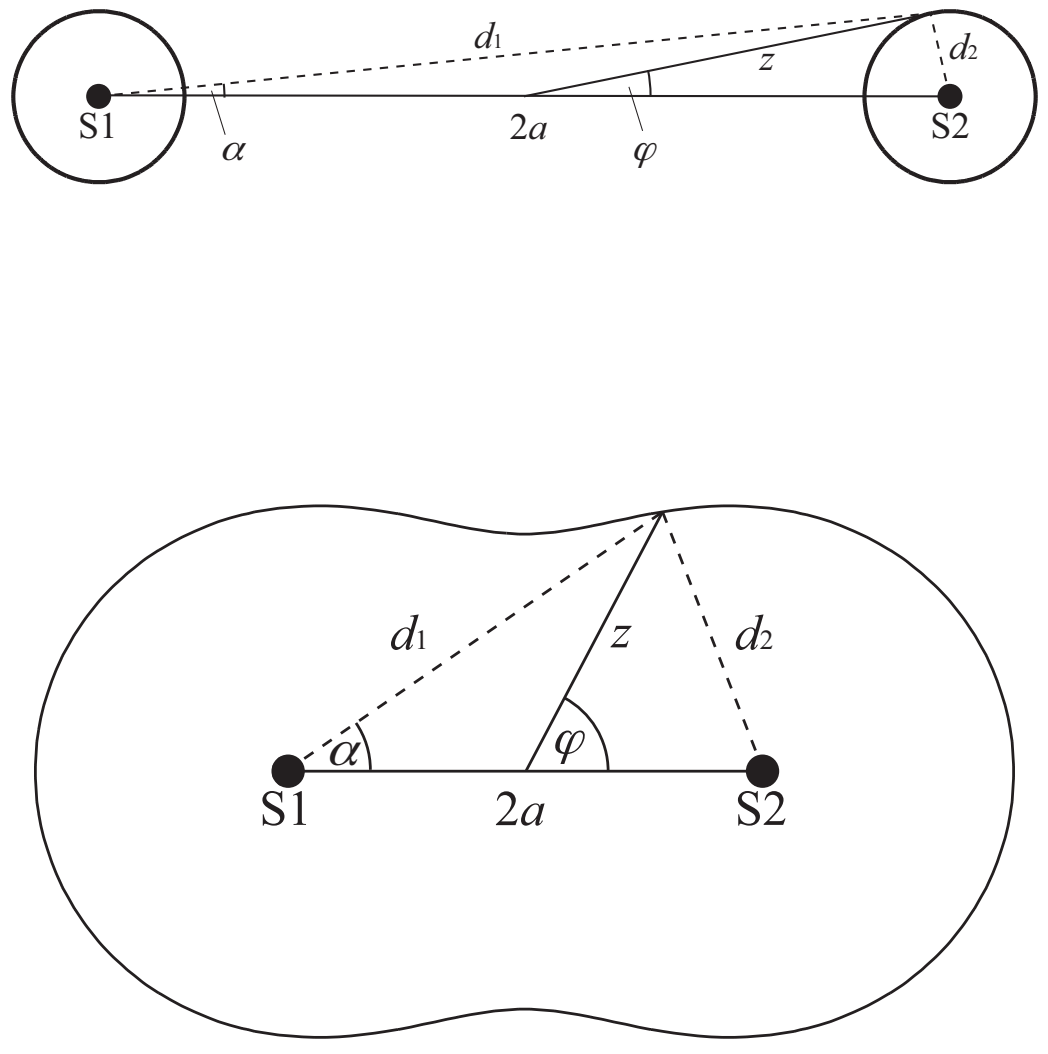

Fig. 1. - Set-up for the mathematical treatment of S-type (top) and P-type (bottom) habitable zones of binary systems as given by the stellar radiative fluxes. Note that the stars S1 and S2 have been depicted as identical for convenience. 


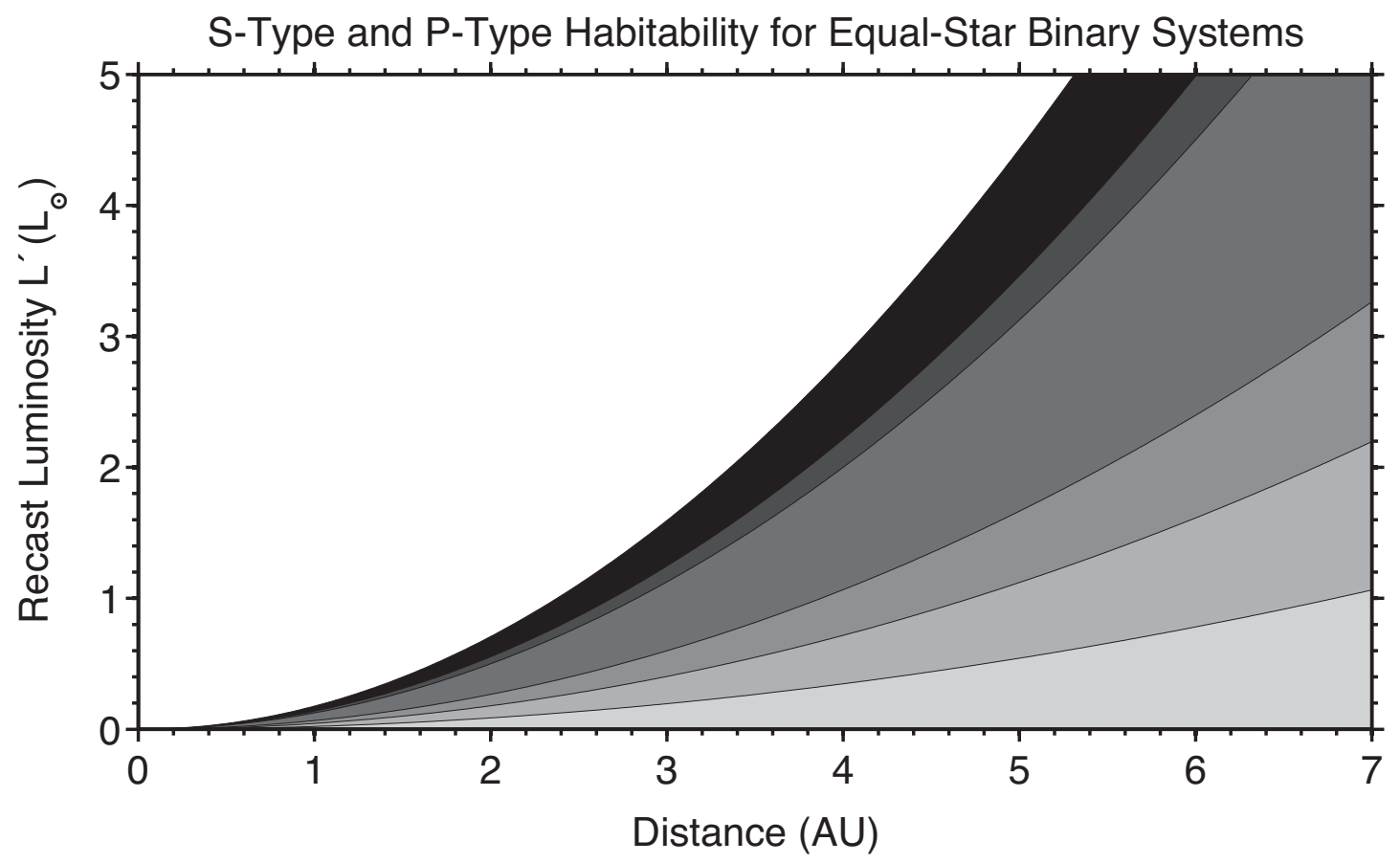

Fig. 2.- Habitability limits for equal-mass binaries regarding S-type and P-type planetary orbits pertaining to the RHZ. The various limits, separated by grayish areas, refer to different values of $s_{\ell}$ given as $0.84,0.95,1.00,1.37,1.67$, and $2.40 \mathrm{AU}$ (from left to right; corresponding to $\ell=1$ to 6 ); see Sect. 2 and 3.2 for details. 

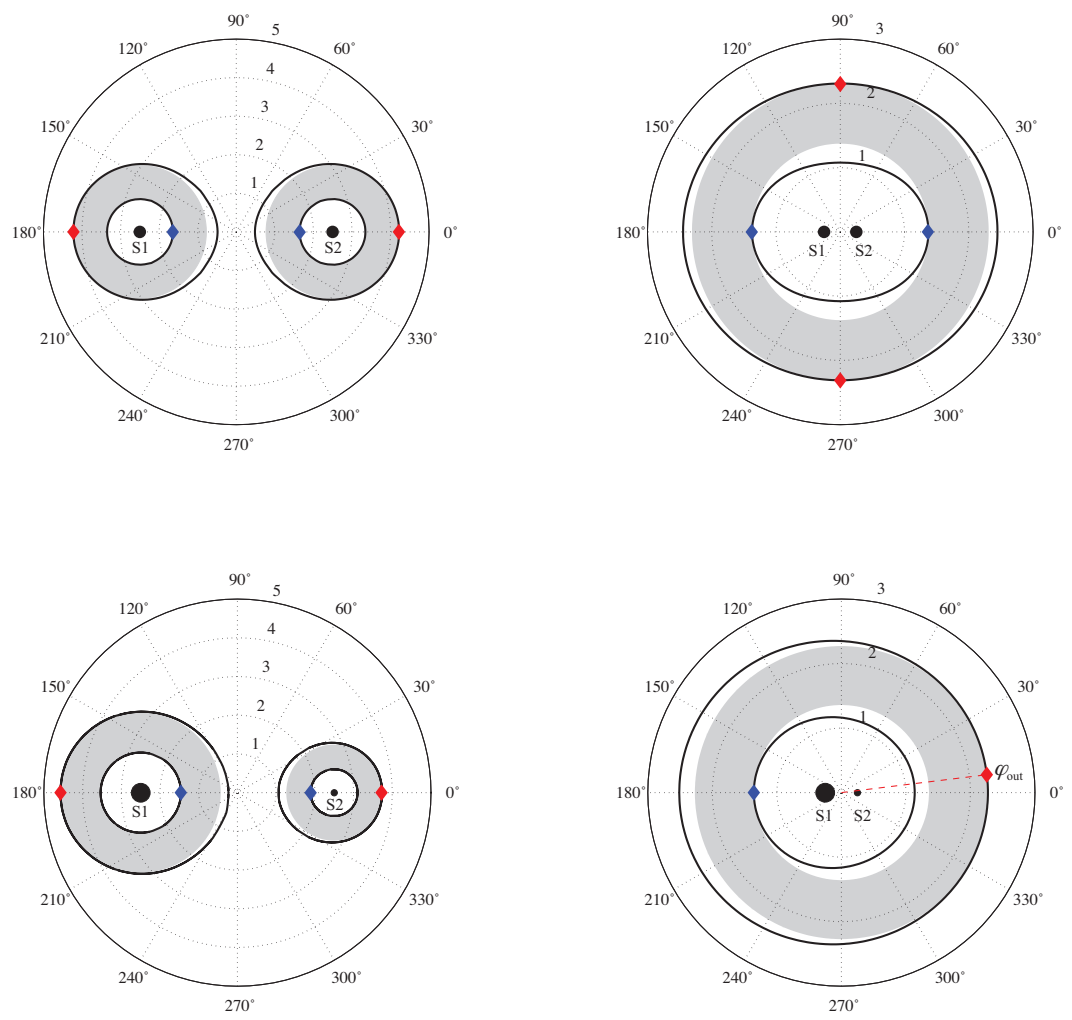

Fig. 3.- Examples of S-type and P-type RHZs for different systems obtained through solutions of the governing fourth-order polynomial equations. The solutions are given in polar coordinates with the radial coordinate depicted in units of AU. The thick solid lines indicate the RHLs, corresponding to the inner and outer limit of habitability based on $s_{\ell}=0.84$ and 1.67 AU, respectively. Note that in the S-type case the RHLs are bended toward the center, whereas in the P-type case they are of elliptical shape. The top row displays systems with $L_{1}=L_{2}=L_{\odot}$, whereas the bottom top row displays systems with $L_{1}=1.5 L_{\odot}$ and $L_{2}=0.5 L_{\odot}$. The left column assumes separation distances $2 a$ of $5.0 \mathrm{AU}$ rendering P-type RHZs, whereas the right column assumes separation distances of $0.5 \mathrm{AU}$ resultant in S-type RHZs. The gray areas indicate the appropriate circular regions (annuli), referred to as RHZs, for each case. The touching points between the RHZs and the inner and outer RHLs (utilized for the definition of the RHZs) are depicted as blue and red diamonds, respectively. They are positioned at angles of $0, \pi / 2, \pi$, and $3 \pi / 2$, except for the outer RHL in case of P-type RHZs for non-equal star binary systems (depicted as $\varphi_{\text {out }}$ ). 


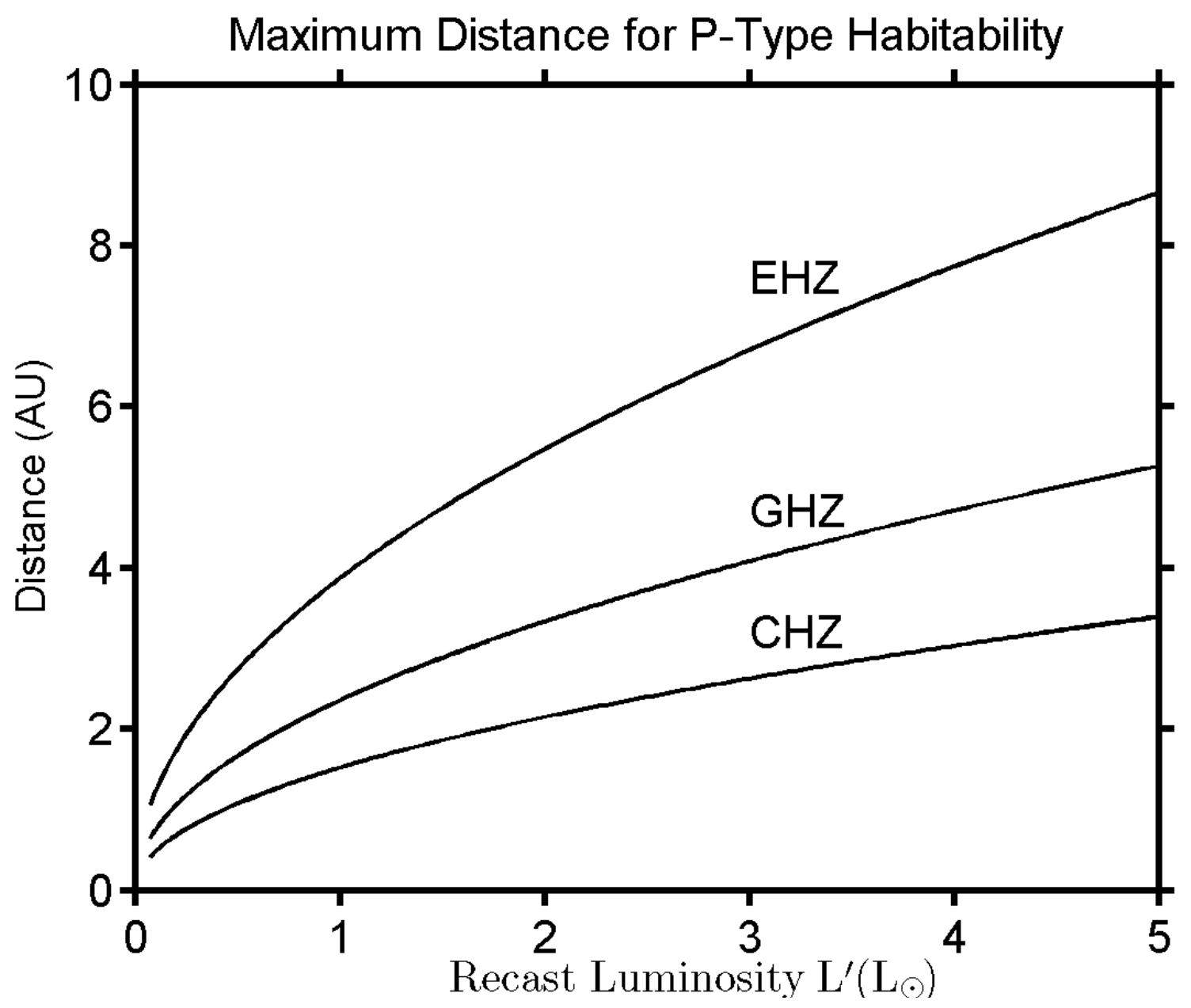

Fig. 4.- Maximum of permissible separation distance $2 a$ in equal-mass binary systems still permitting P-type RHZs (see Eq. 45). Results are given for the CHZ, GHZ, and EHZ. 

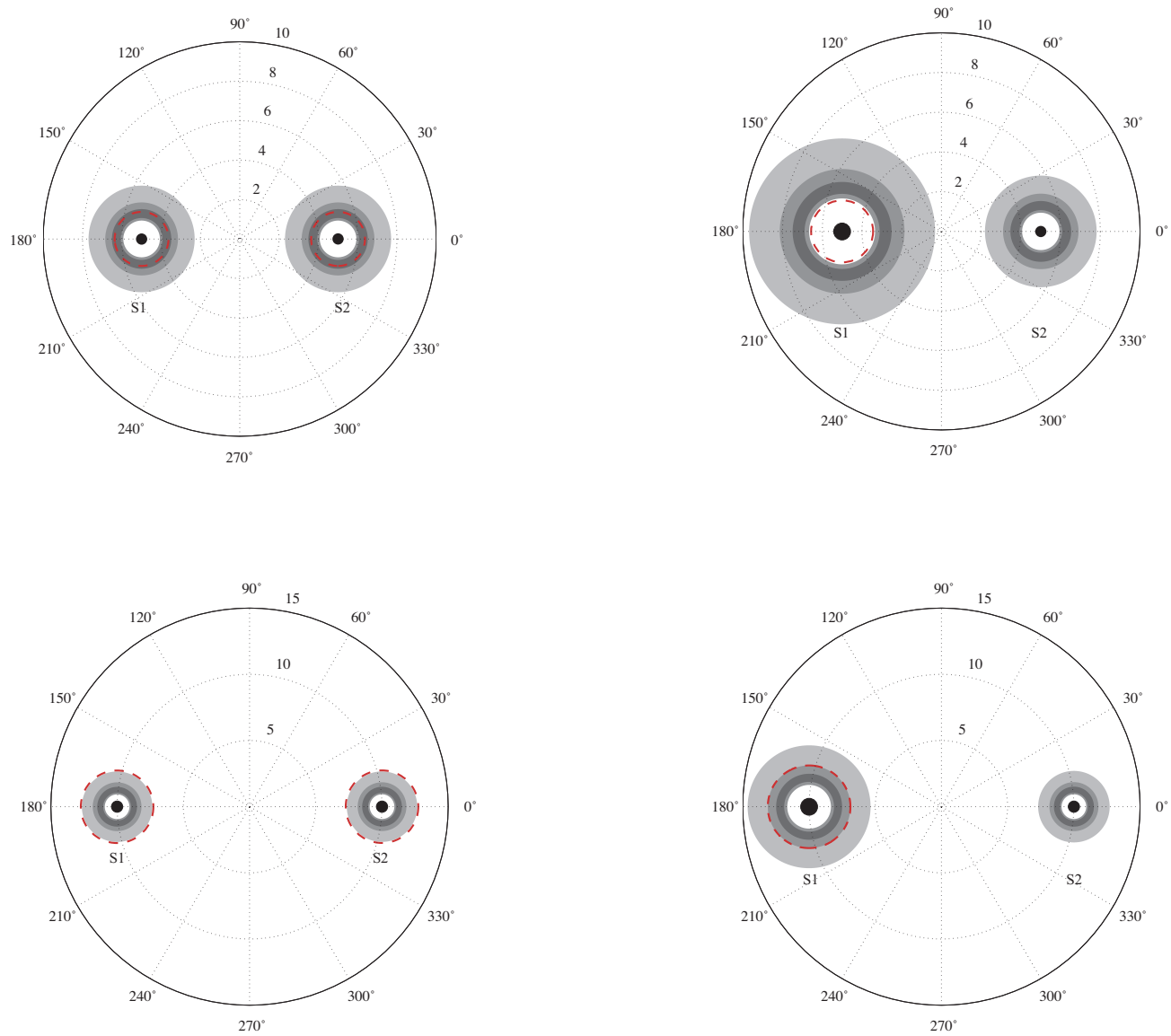

Fig. 5.- Domains of S-type habitability for main-sequence star binary systems. The solutions are given in polar coordinates with the radial coordinate depicted in units of AU. The left column depicts systems with masses of $M_{1}=M_{2}=1.0 M_{\odot}$, whereas the right column depicts systems with masses of $M_{1}=1.5 M_{\odot}$ and $M_{2}=1.0 M_{\odot}$. The separation distances $2 a$ are given as $10 \mathrm{AU}$ (top) and $20 \mathrm{AU}$ (bottom). Results for the conservative, general and extended RHZs are given as dark gray, medium gray, and light gray areas, respectively. The orbital stability limits are indicated by red dashed lines. 

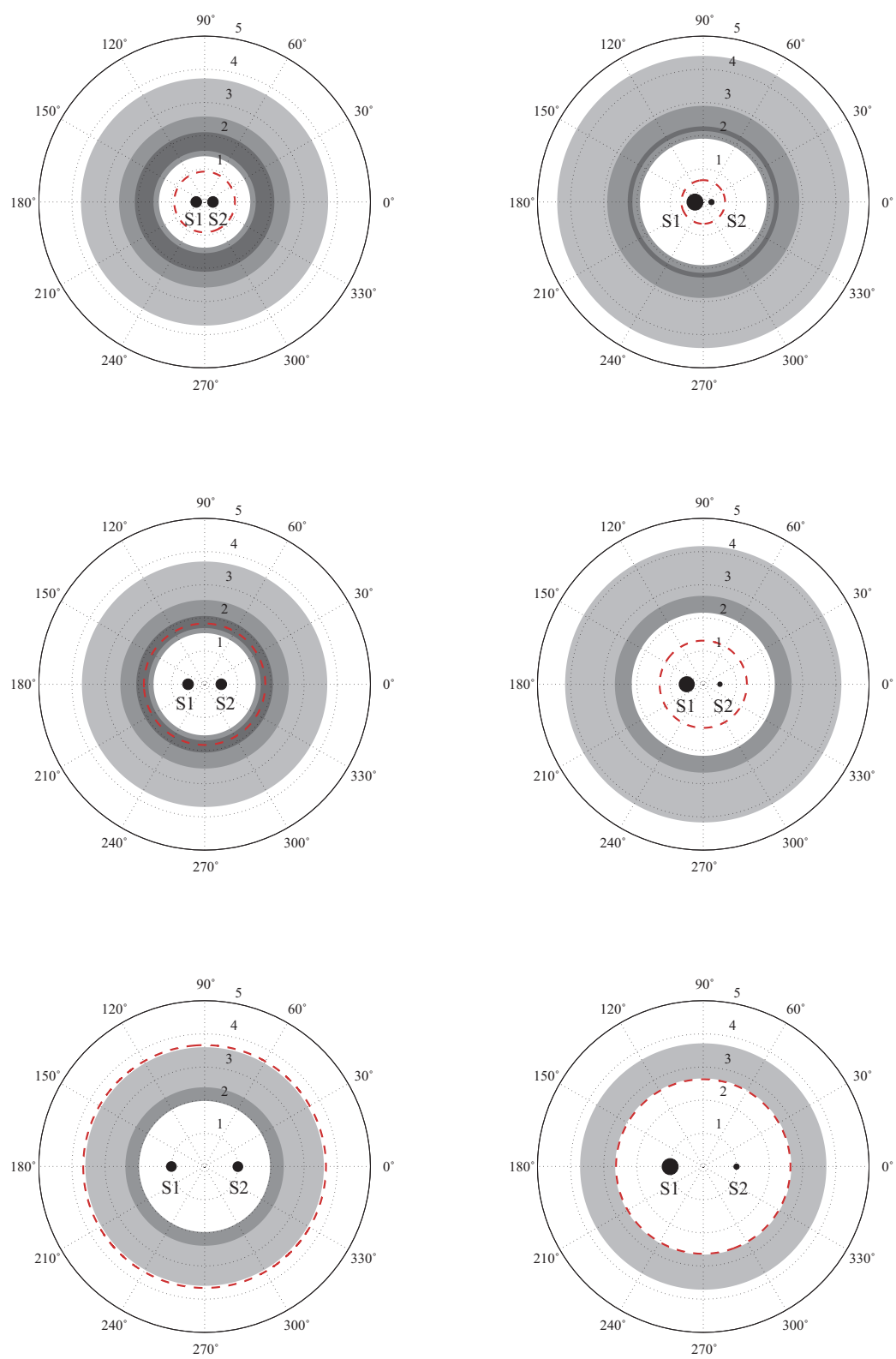

Fig. 6. - Domains of P-type habitability for main-sequence star binary systems. The solutions are given in polar coordinates with the radial coordinate depicted in units of AU. The left column depicts systems with masses of $M_{1}=M_{2}=1.0 M_{\odot}$, whereas the right column depicts systems with masses of $M_{1}=1.5 M_{\odot}$ and $M_{2}=0.5 M_{\odot}$. The separation distances $2 a$ are given as 0.5 AU (top), 1.0 AU (middle), and 2.0 AU (bottom). Results for the conservative, general and extended RHZs are given as dark gray, medium gray, and light gray areas, respectively. The orbital stability limits are indicated by red dashed lines. 

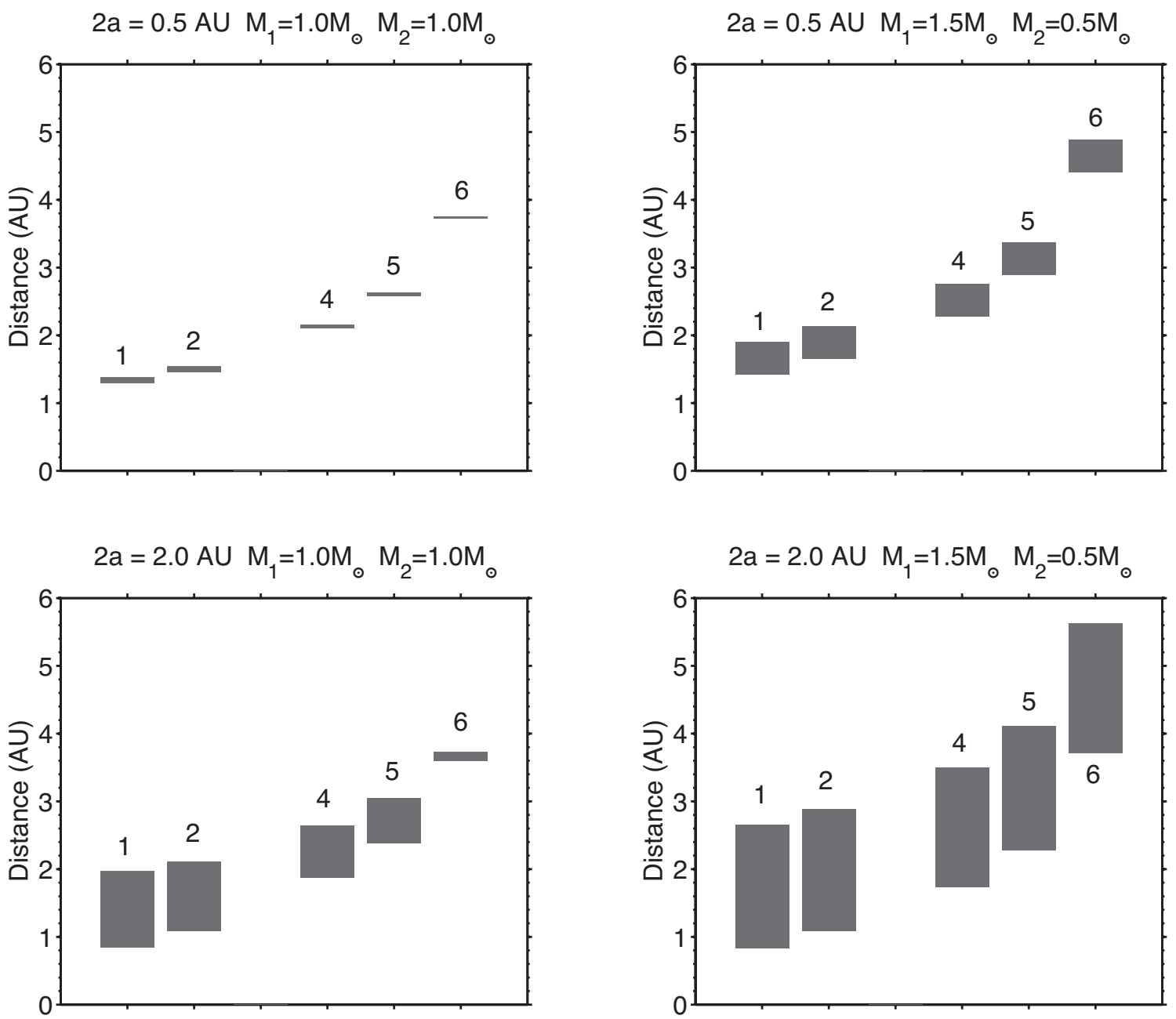

Fig. 7.- Ranges of the inner and outer limits of RHZs also referred to as $\mathrm{RHZ}_{\text {in }}$ and $\mathrm{RHZ}_{\text {out }}$, respectively, pertaining to $s_{\ell}=0.84,0.95,1.37,1.67$, and $2.40 \mathrm{AU}$ (from left to right, with labels $1,2,4,5$, and 6 ). The figure panels depict binary systems with separation distances $2 a$ of $0.5 \mathrm{AU}$ (top) and 2.0 AU (bottom) and with masses of $M_{1}=M_{2}=1.0 M_{\odot}$ (left) and $M_{1}=1.5 M_{\odot}$ and $M_{2}=0.5 M_{\odot}$ (right); see also Tables 6 to 8 for additional information. 

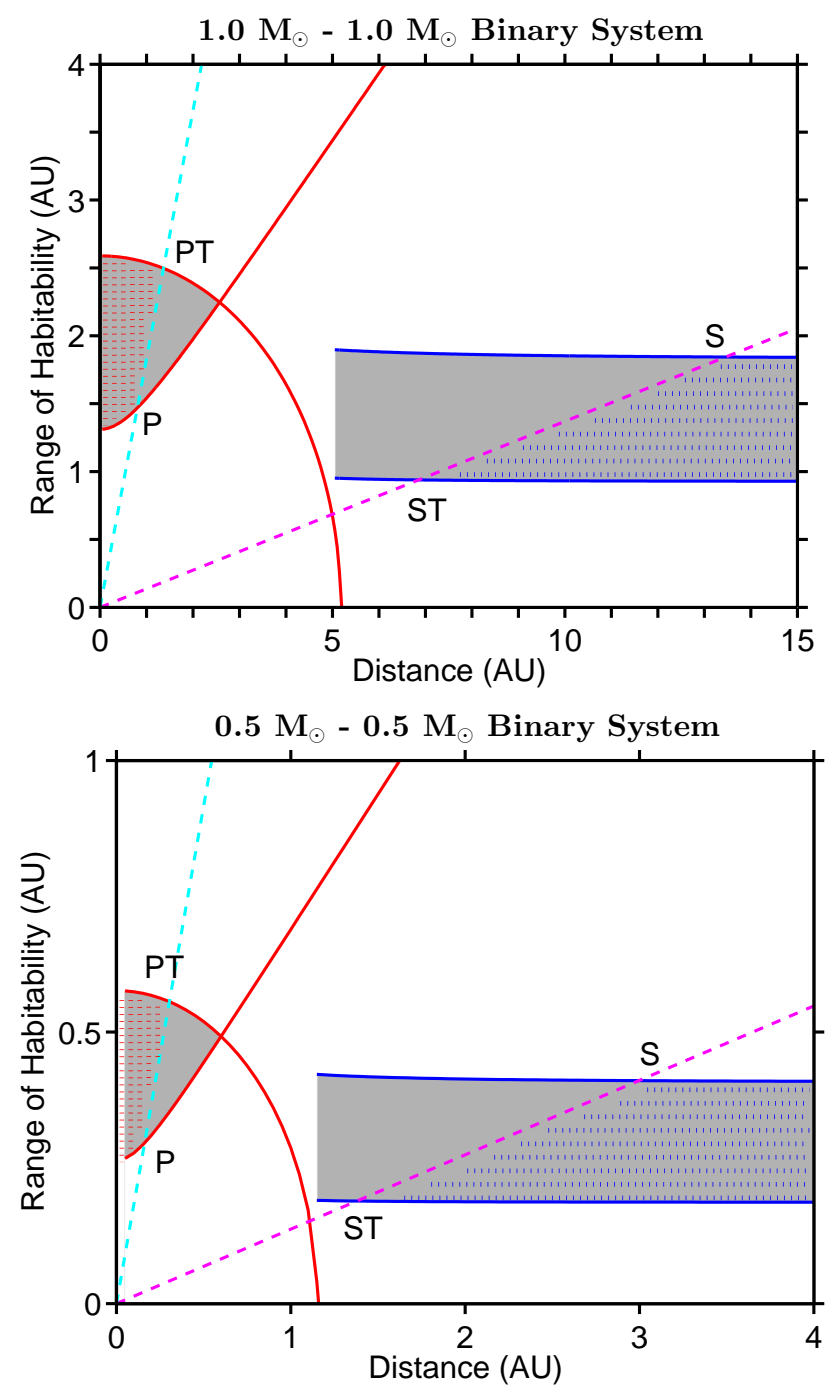

Fig. 8.- Range of habitability in equal-star binary systems with the stellar components given as 1.0 and $0.5 M_{\odot}$, respectively. Results are obtained as function of the binary separation distance $2 a$ pertaining to the GHZ. The two red lines indicate the limits of the P-type RHZ (i.e., $\mathrm{RHZ}_{\text {in }}$ and $\mathrm{RHZ}_{\text {out }}$; see Eqs. (44a) and (44b)), whereas the two blue lines indicate the limits of the S-type RHZ (see Eqs. (41a) and (41b)). The available S-type and P-type RHZs are depicted as grayish areas. The cyan dashed line indicates the P-type orbital stability limit, whereas the violet dashed line indicates the S-type orbital stability limit; see Eqs. (47) and (46), respectively. Note that the P-type orbital stability limit constitutes a lower limit, whereas the S-type orbital stability limit constitutes an upper limit; thus, the available ranges of habitability within the RHZs are indicated as red-hatched and blue-hatched areas, respectively. Hence, P-type habitability is attained in the range beneath the $\mathrm{P}$ intersection point, PT-type habitability between the intersection points $\mathrm{P}$ and PT, ST-type habitability between the intersection points ST and S, and S-type habitability beyond the S intersection point. No habitability is found between the intersection points PT and ST. 

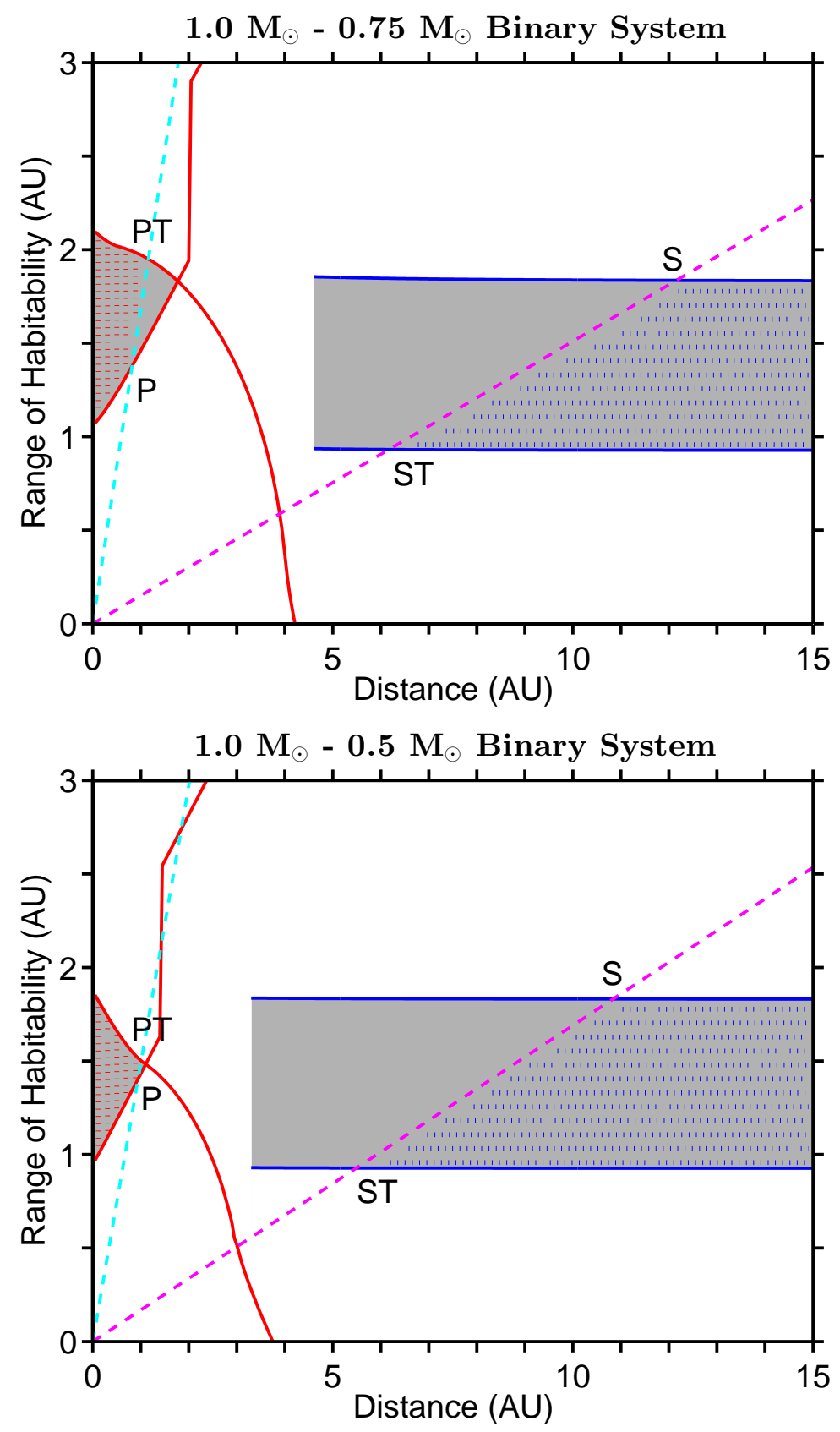

Fig. 9.- Same as Fig. 8, but now for nonequal-star binary systems. The primary is chosen as $1.0 M_{\odot}$, whereas the secondary is chosen as 0.75 and $0.5 M_{\odot}$, respectively. Note that the algebraic solution for P-type $\mathrm{RHZ}_{\text {in }}$ outside the scope of relevance may be ill defined. 


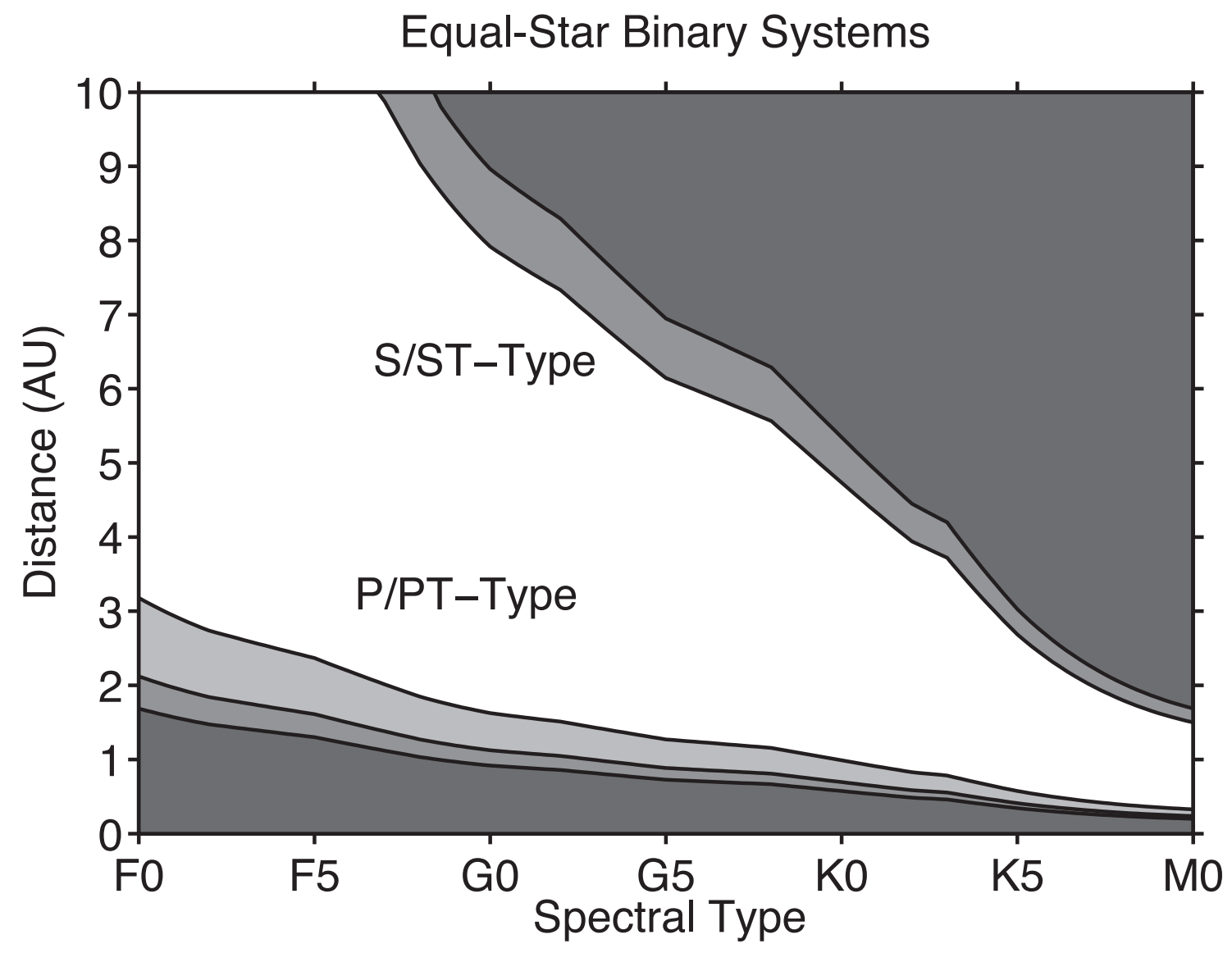

Fig. 10.- Depiction of S/ST-type and P/PT-type habitability based on the joint constraint of planetary orbital stability and the availability of a habitable region provided by the stellar radiative energy fluxes. The domains of the CHZs, GHZs and EHZs are depicted as dark gray, medium gray, and light gray areas, respectively. The results refer to the particular case of equal-mass binaries, i.e., main-sequence stars of identical spectral types. Note that no separate S/ST-type contour is attained for the EHZ, owing to the fact that the inner boundary of the EHZ (i.e., $s_{1}$; see Table 2) agrees with the inner boundary of the GHZ, thus rendering the same mathematical criterion for the inner limit of habitability. For the F0 V, F2 V, and F5 V stars, the S/ST-type contours extend beyond the figure frame. For the GHZ and EHZ, they are given as 15.9, 13.6, and $11.7 \mathrm{AU}$, and for the $\mathrm{CHZ}$, they are given as 18.1, 15.5, and 13.3 AU, respectively. 
Table 1. Stellar Parameters

\begin{tabular}{lccccc}
\hline \hline Sp. Type & $\begin{array}{c}T_{\text {eff }} \\
(\mathrm{K})\end{array}$ & $\begin{array}{c}R_{*} \\
\left(R_{\odot}\right)\end{array}$ & $\begin{array}{c}L_{*} \\
\left(L_{\odot}\right)\end{array}$ & $\begin{array}{c}S_{\text {rel }, *} \\
\cdots\end{array}$ & $\begin{array}{c}M_{*} \\
\left(M_{\odot}\right)\end{array}$ \\
\hline F0 & 7178 & 1.62 & 6.255 & 1.145 & 1.60 \\
F2 & 6909 & 1.48 & 4.481 & 1.113 & 1.52 \\
F5 & 6528 & 1.40 & 3.196 & 1.072 & 1.40 \\
F8 & 6160 & 1.20 & 1.862 & 1.037 & 1.19 \\
G0 & 5943 & 1.12 & 1.405 & 1.019 & 1.05 \\
G2 & 5811 & 1.08 & 1.194 & 1.009 & 0.99 \\
G5 & 5657 & 0.95 & 0.830 & 0.997 & 0.91 \\
G8 & 5486 & 0.91 & 0.673 & 0.985 & 0.84 \\
K0 & 5282 & 0.83 & 0.481 & 0.971 & 0.79 \\
K2 & 5055 & 0.75 & 0.330 & 0.957 & 0.74 \\
K5 & 4487 & 0.64 & 0.149 & 0.926 & 0.67 \\
K8 & 4006 & 0.53 & 0.066 & 0.905 & 0.58 \\
M0 & 3850 & 0.48 & 0.045 & 0.900 & 0.51 \\
\hline
\end{tabular}

Note. $-S_{\text {rel, } *}$ is calculated for $s_{\ell}=s_{3}$. 
Table 2. Habitability Limits for the Sun

\begin{tabular}{|c|c|c|c|}
\hline $\begin{array}{l}\ell \\
\ldots\end{array}$ & $\begin{array}{c}s_{\ell} \\
(\mathrm{AU})\end{array}$ & $\begin{array}{c}\text { HZ Limit } \\
\ldots\end{array}$ & $\begin{array}{c}\text { Description } \\
\ldots\end{array}$ \\
\hline 1 & 0.84 & GHZ / EHZ & Runaway greenhouse effect \\
\hline 2 & 0.95 & $\mathrm{CHZ}$ & Start of water loss \\
\hline 3 & 1.00 & $\ldots$ & Earth-equivalent position \\
\hline 4 & 1.37 & $\mathrm{CHZ}$ & First $\mathrm{CO}_{2}$ condensation \\
\hline 5 & 1.67 & GHZ & Maximum greenhouse effect, no clouds \\
\hline 6 & 2.40 & EHZ & Maximum greenhouse effect, $100 \%$ clouds \\
\hline
\end{tabular}

Note. - See text for references. 
Table 3. Habitable Zones of Single Main-Sequence Stars

\begin{tabular}{lcccccc}
\hline \hline Sp. Type & \multicolumn{5}{c}{ Habitable Zone } \\
\hline$\ldots$ & $\begin{array}{c}\mathrm{HZ}\left(s_{1}\right) \\
(\mathrm{AU})\end{array}$ & $\begin{array}{c}\mathrm{HZ}\left(s_{2}\right) \\
(\mathrm{AU})\end{array}$ & $\begin{array}{c}\mathrm{HZ}\left(s_{3}\right) \\
(\mathrm{AU})\end{array}$ & $\begin{array}{c}\mathrm{HZ}\left(s_{4}\right) \\
(\mathrm{AU})\end{array}$ & $\begin{array}{c}\mathrm{HZ}\left(s_{5}\right) \\
(\mathrm{AU})\end{array}$ & $\begin{array}{c}\mathrm{HZ}\left(s_{6}\right) \\
(\mathrm{AU})\end{array}$ \\
\hline$\ldots$ & 1.98 & 2.25 & 2.33 & 2.91 & 3.66 & 5.49 \\
F0 & 1.70 & 1.93 & 2.00 & 2.54 & 3.18 & 4.72 \\
F2 & 1.46 & 1.65 & 1.72 & 2.24 & 2.78 & 4.08 \\
F5 & 1.13 & 1.28 & 1.34 & 1.78 & 2.19 & 3.19 \\
F8 & 0.99 & 1.12 & 1.17 & 1.58 & 1.94 & 2.80 \\
$\mathrm{G} 0$ & 0.91 & 1.03 & 1.09 & 1.48 & 1.81 & 2.61 \\
$\mathrm{G} 2$ & 0.77 & 0.87 & 0.91 & 1.25 & 1.53 & 2.19 \\
$\mathrm{G} 5$ & 0.69 & 0.78 & 0.83 & 1.15 & 1.39 & 1.99 \\
$\mathrm{G} 8$ & 0.59 & 0.67 & 0.71 & 0.99 & 1.20 & 1.71 \\
K0 & 0.49 & 0.55 & 0.59 & 0.84 & 1.01 & 1.43 \\
$\mathrm{~K} 2$ & 0.34 & 0.38 & 0.40 & 0.59 & 0.71 & 0.99 \\
K5 & 0.22 & 0.25 & 0.27 & 0.41 & 0.49 & 0.67 \\
K8 & 0.19 & 0.21 & 0.23 & 0.35 & 0.41 & 0.56 \\
M0 & & & & & & \\
\hline
\end{tabular}


Table 4. Recast Stellar Luminosity

\begin{tabular}{lccccccc}
\hline \hline Sp. Type & $\begin{array}{c}T_{\text {eff }} \\
(\mathrm{K})\end{array}$ & $\begin{array}{c}L_{i 1}^{\prime} \\
\left(L_{\odot}\right)\end{array}$ & $\begin{array}{c}L_{i 2}^{\prime} \\
\left(L_{\odot}\right)\end{array}$ & $\begin{array}{c}L_{i 3}^{\prime} \\
\left(L_{\odot}\right)\end{array}$ & $\begin{array}{c}L_{i 4}^{\prime} \\
\left(L_{\odot}\right)\end{array}$ & $\begin{array}{c}L_{i 5}^{\prime} \\
\left(L_{\odot}\right)\end{array}$ & $\begin{array}{c}L_{i 6}^{\prime} \\
\left(L_{\odot}\right)\end{array}$ \\
\hline F0 & 7178 & 5.545 & 5.625 & 5.464 & 4.509 & 4.801 & 5.223 \\
F2 & 6909 & 4.075 & 4.121 & 4.027 & 3.445 & 3.621 & 3.873 \\
F5 & 6528 & 3.005 & 3.027 & 2.981 & 2.681 & 2.770 & 2.897 \\
F8 & 6160 & 1.802 & 1.809 & 1.794 & 1.692 & 1.722 & 1.764 \\
G0 & 5943 & 1.382 & 1.384 & 1.379 & 1.337 & 1.349 & 1.366 \\
G2 & 5811 & 1.185 & 1.186 & 1.184 & 1.168 & 1.172 & 1.179 \\
G5 & 5657 & 0.832 & 0.832 & 0.832 & 0.837 & 0.836 & 0.834 \\
G8 & 5486 & 0.683 & 0.682 & 0.684 & 0.703 & 0.697 & 0.690 \\
K0 & 5282 & 0.494 & 0.493 & 0.496 & 0.523 & 0.515 & 0.505 \\
K2 & 5055 & 0.343 & 0.341 & 0.345 & 0.374 & 0.366 & 0.354 \\
K5 & 4487 & 0.150 & 0.149 & 0.151 & 0.177 & 0.170 & 0.161 \\
K8 & 4006 & 0.071 & 0.071 & 0.072 & 0.089 & 0.085 & 0.079 \\
M0 & 3850 & 0.050 & 0.049 & 0.051 & 0.064 & 0.060 & 0.055 \\
\hline
\end{tabular}

Note. - $L_{i \ell}^{\prime}$ denotes general binaries with $i=1,2$ for star S1 and $\mathrm{S} 2$, respectively, with $\ell=1$ to 6 . 
Table 5. Target List, Sections 5 and 6

\begin{tabular}{cccccc}
\hline \hline $\begin{array}{l}M_{*} \\
\left(M_{\odot}\right)\end{array}$ & $\begin{array}{c}\text { Spectral Type } \\
\ldots\end{array}$ & $\begin{array}{c}T_{\text {eff }} \\
(\mathrm{K})\end{array}$ & $\begin{array}{c}L_{*} \\
\left(L_{\odot}\right)\end{array}$ & $\begin{array}{c}L_{i 3}^{\prime} \\
\left(L_{\odot}\right)\end{array}$ & $\begin{array}{c}\mathrm{HZ}\left(s_{3}\right) \\
(\mathrm{AU})\end{array}$ \\
\hline 1.50 & $\mathrm{~F} 2 \mathrm{~V}$ & 6842 & 4.233 & 3.830 & 1.96 \\
1.25 & $\mathrm{~F} 7 \mathrm{~V}$ & 6256 & 2.173 & 2.078 & 1.44 \\
1.00 & $\mathrm{G} 2 \mathrm{~V}$ & 5833 & 1.228 & 1.216 & 1.10 \\
0.75 & $\mathrm{~K} 2 \mathrm{~V}$ & 5100 & 0.357 & 0.372 & 0.61 \\
0.50 & $\mathrm{M} 0 \mathrm{~V}$ & 3857 & 0.045 & 0.050 & 0.22 \\
\hline
\end{tabular}

Note. - $L_{i 3}^{\prime}$ denotes general binaries with $i=1,2$ for star S1 and S2, respectively. 
Table 6. P-Type Stellar Habitability; $2 a=0.5$ AU

\begin{tabular}{|c|c|c|c|c|}
\hline \multicolumn{2}{|c|}{$\mathrm{HZ}$} & \multicolumn{3}{|c|}{ Binary System Data } \\
\hline $\begin{array}{l}\ell \\
\ldots\end{array}$ & $\begin{array}{c}s_{\ell} \\
(\mathrm{AU})\end{array}$ & $\begin{array}{c}\mathrm{RHZ}_{\mathrm{in}} \\
(\mathrm{AU})\end{array}$ & $\begin{array}{c}\mathrm{RHZ}_{\text {out }} \\
(\mathrm{AU})\end{array}$ & $\begin{array}{c}a_{\mathrm{cr}} \\
(\mathrm{AU})\end{array}$ \\
\hline \multicolumn{5}{|c|}{ Model: $M_{1}=1.0 M_{\odot}, M_{2}=1.0 M_{\odot}$} \\
\hline 1 & 0.84 & 1.29 & 1.38 & 0.92 \\
\hline 2 & 0.95 & 1.46 & 1.54 & 0.92 \\
\hline 3 & 1.00 & 1.54 & 1.62 & 0.92 \\
\hline 4 & 1.37 & 2.10 & 2.16 & 0.92 \\
\hline 5 & 1.67 & 2.58 & 2.62 & 0.92 \\
\hline 6 & 2.40 & 3.72 & 3.76 & 0.92 \\
\hline \multicolumn{5}{|c|}{ Model: $M_{1}=1.25 M_{\odot}, M_{2}=0.75 M_{\odot}$} \\
\hline 1 & 0.84 & 1.19 & 1.52 & 0.79 \\
\hline 2 & 0.95 & 1.36 & 1.69 & 0.79 \\
\hline 3 & 1.00 & 1.45 & 1.79 & 0.79 \\
\hline 4 & 1.37 & 1.96 & 2.28 & 0.79 \\
\hline 5 & 1.67 & 2.43 & 2.76 & 0.79 \\
\hline 6 & 2.40 & 3.58 & 3.91 & 0.79 \\
\hline \multicolumn{5}{|c|}{ Model: $M_{1}=1.5 M_{\odot}, M_{2}=0.5 M_{\odot}$} \\
\hline 1 & 0.84 & 1.42 & 1.91 & 0.66 \\
\hline 2 & 0.95 & 1.65 & 2.14 & 0.66 \\
\hline 3 & 1.00 & 1.73 & 2.22 & 0.66 \\
\hline 4 & 1.37 & 2.28 & 2.76 & 0.66 \\
\hline 5 & 1.67 & 2.90 & 3.38 & 0.66 \\
\hline 6 & 2.40 & 4.40 & 4.89 & 0.66 \\
\hline
\end{tabular}


Table 7. P-Type Stellar Habitability; $2 a=1.0$ AU

\begin{tabular}{|c|c|c|c|c|}
\hline \multicolumn{2}{|c|}{$\mathrm{HZ}$} & \multicolumn{3}{|c|}{ Binary System Data } \\
\hline $\begin{array}{l}\ell \\
\ldots\end{array}$ & $\begin{array}{c}s_{\ell} \\
(\mathrm{AU})\end{array}$ & $\begin{array}{c}\mathrm{RHZ}_{\mathrm{in}} \\
(\mathrm{AU})\end{array}$ & $\begin{array}{c}\mathrm{RHZ}_{\text {out }} \\
(\mathrm{AU})\end{array}$ & $\begin{array}{c}a_{\mathrm{cr}} \\
(\mathrm{AU})\end{array}$ \\
\hline \multicolumn{5}{|c|}{ Model: $M_{1}=1.0 M_{\odot}, M_{2}=1.0 M_{\odot}$} \\
\hline 1 & 0.84 & 1.21 & 1.54 & 1.83 \\
\hline 2 & 0.95 & 1.40 & 1.69 & 1.83 \\
\hline 3 & 1.00 & 1.48 & 1.76 & 1.83 \\
\hline 4 & 1.37 & 2.06 & 2.28 & 1.83 \\
\hline 5 & 1.67 & 2.54 & 2.72 & 1.83 \\
\hline 6 & 2.40 & 3.70 & 3.83 & 1.83 \\
\hline \multicolumn{5}{|c|}{ Model: $M_{1}=1.25 M_{\odot}, M_{2}=0.75 M_{\odot}$} \\
\hline 1 & 0.84 & 1.11 & 1.75 & 1.57 \\
\hline 2 & 0.95 & 1.29 & 1.92 & 1.57 \\
\hline 3 & 1.00 & 1.38 & 2.02 & 1.57 \\
\hline 4 & 1.37 & 1.90 & 2.49 & 1.57 \\
\hline 5 & 1.67 & 2.35 & 2.96 & 1.57 \\
\hline 6 & 2.40 & 3.46 & 4.12 & 1.57 \\
\hline \multicolumn{5}{|c|}{ Model: $M_{1}=1.5 M_{\odot}, M_{2}=0.5 M_{\odot}$} \\
\hline 1 & 0.84 & 1.21 & 2.16 & 1.32 \\
\hline 2 & 0.95 & 1.43 & 2.38 & 1.32 \\
\hline 3 & 1.00 & 1.51 & 2.47 & 1.32 \\
\hline 4 & 1.37 & 2.05 & 3.00 & 1.32 \\
\hline 5 & 1.67 & 2.66 & 3.62 & 1.32 \\
\hline 6 & 2.40 & 4.17 & 5.13 & 1.32 \\
\hline
\end{tabular}


Table 8. P-Type Stellar Habitability; $2 a=2.0$ AU

\begin{tabular}{|c|c|c|c|c|}
\hline \multicolumn{2}{|c|}{$\mathrm{HZ}$} & \multicolumn{3}{|c|}{ Binary System Data } \\
\hline $\begin{array}{l}\ell \\
\ldots\end{array}$ & $\begin{array}{c}s_{\ell} \\
(\mathrm{AU})\end{array}$ & $\begin{array}{c}\mathrm{RHZ}_{\text {in }} \\
(\mathrm{AU})\end{array}$ & $\begin{array}{c}\mathrm{RHZ}_{\text {out }} \\
(\mathrm{AU})\end{array}$ & $\begin{array}{c}a_{\mathrm{cr}} \\
(\mathrm{AU})\end{array}$ \\
\hline \multicolumn{5}{|c|}{ Model: $M_{1}=1.0 M_{\odot}, M_{2}=1.0 M_{\odot}$} \\
\hline 1 & 0.84 & 0.85 & 1.98 & 3.66 \\
\hline 2 & 0.95 & 1.10 & 2.11 & 3.66 \\
\hline 3 & 1.00 & 1.20 & 2.18 & 3.66 \\
\hline 4 & 1.37 & 1.87 & 2.64 & 3.66 \\
\hline 5 & 1.67 & 2.39 & 3.05 & 3.66 \\
\hline 6 & 2.40 & 3.60 & 4.09 & 3.66 \\
\hline \multicolumn{5}{|c|}{ Model: $M_{1}=1.25 M_{\odot}, M_{2}=0.75 M_{\odot}$} \\
\hline 1 & 0.84 & 0.70 & 2.23 & 3.15 \\
\hline 2 & 0.95 & 0.95 & 2.40 & 3.15 \\
\hline 3 & 1.00 & 1.07 & 2.50 & 3.15 \\
\hline 4 & 1.37 & 1.69 & 2.95 & 3.15 \\
\hline 5 & 1.67 & 2.18 & 3.42 & 3.15 \\
\hline 6 & 2.40 & 3.32 & 4.55 & 3.15 \\
\hline \multicolumn{5}{|c|}{ Model: $M_{1}=1.5 M_{\odot}, M_{2}=0.5 M_{\odot}$} \\
\hline 1 & 0.84 & 0.83 & 2.66 & 2.63 \\
\hline 2 & 0.95 & 1.09 & 2.88 & 2.63 \\
\hline 3 & 1.00 & 1.18 & 2.96 & 2.63 \\
\hline 4 & 1.37 & 1.74 & 3.50 & 2.63 \\
\hline 5 & 1.67 & 2.28 & 4.12 & 2.63 \\
\hline 6 & 2.40 & 3.71 & 5.63 & 2.63 \\
\hline
\end{tabular}


Table 9. Maximum Binary Separation Distance $2 a$ Permitting P-type RHZs

\begin{tabular}{|c|c|c|c|c|c|}
\hline \multirow{2}{*}{$\begin{array}{l}\text { Primary } \\
\ldots\end{array}$} & \multicolumn{5}{|c|}{ Secondary } \\
\hline & $M_{2}=1.5 M_{\odot}$ & $1.25 M_{\odot}$ & $1.0 M_{\odot}$ & $0.75 M_{\odot}$ & $0.5 M_{\odot}$ \\
\hline$M_{1}$ & \multicolumn{5}{|c|}{ Maximum Binary Separation Distance } \\
\hline$\left(M_{\odot}\right)$ & $(\mathrm{AU})$ & $(\mathrm{AU})$ & $(\mathrm{AU})$ & $(\mathrm{AU})$ & $(\mathrm{AU})$ \\
\hline \multicolumn{6}{|c|}{$\mathrm{CHZ}$ given as $\left(s_{2}, s_{4}\right)$} \\
\hline 1.5 & 2.47 & 1.90 & 1.50 & 0.87 & 0.65 \\
\hline 1.25 & $\ldots$ & 2.01 & 1.61 & 0.96 & 0.58 \\
\hline 1.0 & $\ldots$ & $\ldots$ & 1.64 & 1.01 & 0.53 \\
\hline 0.75 & $\ldots$ & $\ldots$ & $\ldots$ & 1.00 & 0.47 \\
\hline 0.5 & $\ldots$ & $\ldots$ & $\ldots$ & $\ldots$ & 0.43 \\
\hline \multicolumn{6}{|c|}{ GHZ given as $\left(s_{1}, s_{5}\right)$} \\
\hline 1.5 & 4.25 & 3.48 & 2.95 & 2.14 & 1.55 \\
\hline 1.25 & $\ldots$ & 3.26 & 2.74 & 1.93 & 1.27 \\
\hline 1.0 & $\ldots$ & $\ldots$ & 2.57 & 1.77 & 1.10 \\
\hline 0.75 & $\ldots$ & $\ldots$ & $\ldots$ & 1.50 & 0.84 \\
\hline 0.5 & $\ldots$ & $\ldots$ & $\ldots$ & $\ldots$ & 0.60 \\
\hline \multicolumn{6}{|c|}{ EHZ given as $\left(s_{1}, s_{6}\right)$} \\
\hline 1.5 & 7.38 & 7.15 & 5.96 & 4.43 & 3.29 \\
\hline 1.25 & $\ldots$ & 5.50 & 5.48 & 3.80 & 2.64 \\
\hline 1.0 & $\ldots$ & $\ldots$ & 4.25 & 3.41 & 2.19 \\
\hline 0.75 & $\ldots$ & $\ldots$ & $\ldots$ & 2.40 & 1.55 \\
\hline 0.5 & $\ldots$ & $\ldots$ & $\ldots$ & $\ldots$ & 0.92 \\
\hline
\end{tabular}




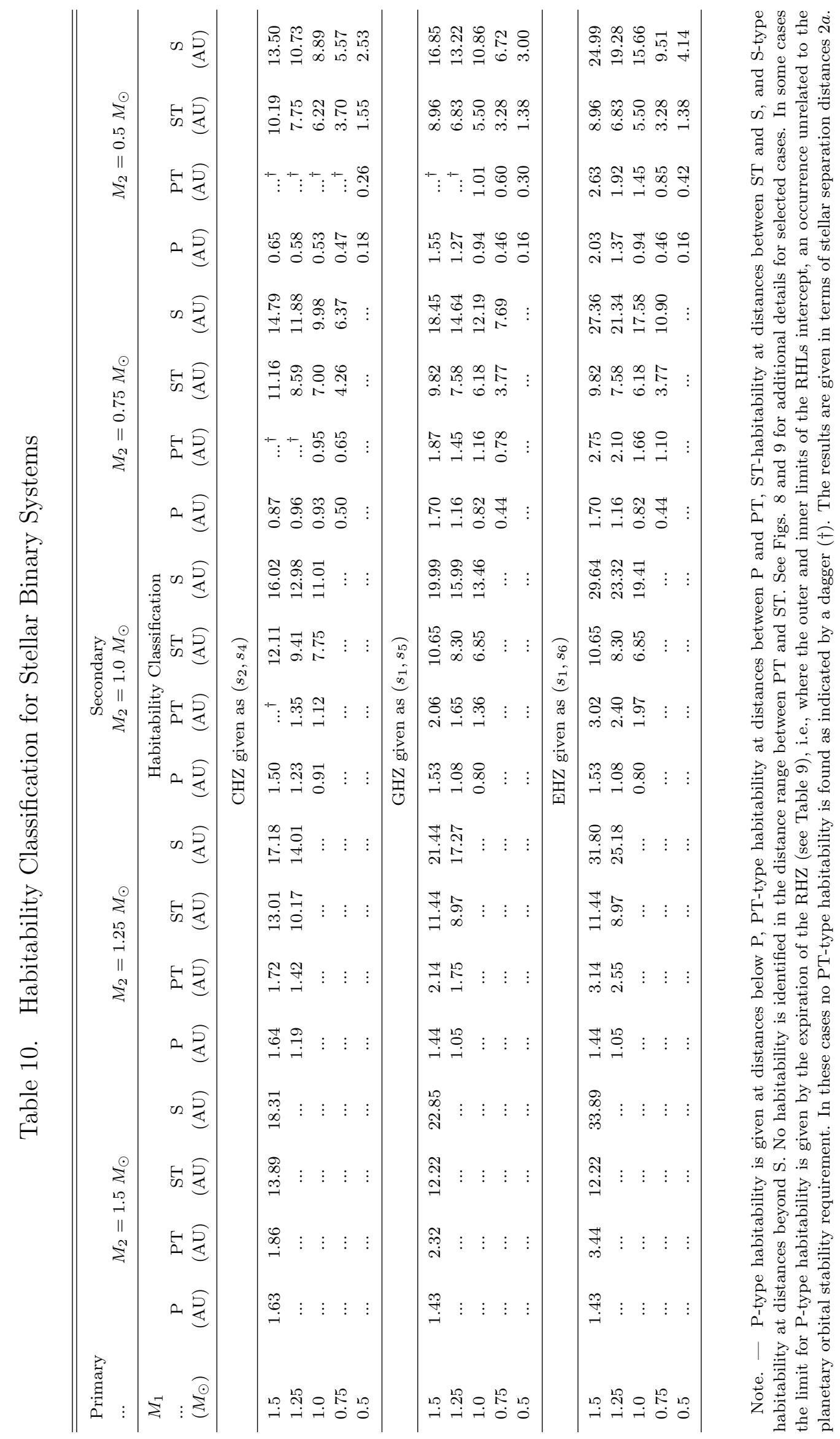

